Research Article

\title{
Phytochemical Analysis Using UPLC-MS/MS Combined with Network Pharmacology Methods to Explore the Biomarkers for the Quality Control of Lingguizhugan Decoction
}

\author{
Baolin Li $\mathbb{D},{ }^{1,2,3}$ Shuaishuai Fan $\mathbb{D}^{1},{ }^{1}$ Jingnan Hu $\mathbb{D}^{1},{ }^{1}$ Yongben Ma $\mathbb{D}^{1},{ }^{1}$ Yu Feng $\mathbb{D},{ }^{1}$ \\ Fengxia Wang $\mathbb{D}^{1}$, Xinguo Wang $₫$, ${ }^{1,2,3}$ and Liying Niu $\rrbracket^{1,2,3}$ \\ ${ }^{1}$ College of Integrative Medicine, Hebei University of Chinese Medicine, \\ Shijiazhuang 050091, China \\ ${ }^{2}$ Hebei Traditional Chinese Medicine Formula Granule Engineering \& Technology Innovate Center, Shijiazhuang 050091, China \\ ${ }^{3}$ Quality Evaluation \& Standardization Hebei Province Engineering Research Center of Traditional Chinese Medicine, \\ Shijiazhuang 050091, China
}

Correspondence should be addressed to Xinguo Wang; wangxinguozy@163.com and Liying Niu; niuliyingyy@163.com

Received 17 September 2021; Accepted 23 November 2021; Published 22 December 2021

Academic Editor: Nahla S. Abdel Azim

Copyright $\odot 2021$ Baolin Li et al. This is an open access article distributed under the Creative Commons Attribution License, which permits unrestricted use, distribution, and reproduction in any medium, provided the original work is properly cited.

\begin{abstract}
As a classic TCM prescription, LGZG has been widely used in clinical prevention and treatment of heart failure, nonalcoholic fatty liver, and hyperlipidemia. However, there are few studies on chemical components in recent years, and the basis of quality evaluation is not sufficient. This study was to find the active ingredients of the Lingguizhugan decoction using UPLC-MS/MS and network pharmacology. By comparing the retention time and MS dates of the reference and self-building database, the cleavage rules of chemical composition whose mass errors are less than 1 ppm(FL less than 3 ppm) are analyzed. On this basis, a network pharmacology method was used to find biomarkers for quantitative analysis. The results show that 149 compounds were preliminaries identified or inferred, including 63 flavonoids, 30 triterpenes, 22 phenylpropanoids, 13 organic acids, 6 lactones, 5 alkaloids, 4 anthraquinones, and 6 other compounds. According to the network pharmacology results, 20 chemical constituents were selected as the biomarkers, which were determined simultaneously for the first time, including poricoic acid A, poricoic acid B, glycyrrhizic acid, glycyrrhetinic acid, liquiritin, isoliquiritin, liquiritigenin, isoliquiritin apioside, cinnamic acid, caffeic acid, neochlorogenic acid, chlorogenic acid, cryptochlorogenic acid, isochlorogenic acid A, B, and C, atractylenolide I, II, and III, and coumarin. The methodological results show that the linearity, stability, precision, repeatability, and recovery of the method are satisfactory. Therefore, a comprehensive quality assessment system for LGZG was established on the basis of a systematic study of chemical substances and network pharmacology, which provided an important reference for the foundation of pharmacological action and its mechanics.
\end{abstract}

\section{Introduction}

Lingguizhugan decoction (LGZG), coming from Zhang Zhongjing's Synopsis of Preions of the Golden Chamber, has already been listed as the first batch of "The Catalogue of ancient classical prescription" by the National Administration of Traditional Chinese Medicine (TCM) in 2018. LGZG is prepared by four different herbal medicines, namely Fuling (the dry sclerotia of Poria cocos (Schw.), FL), Guizhi (the dry twigs of Cinnamomum cassia Presl, GZ),
Baizhu (the dry rhizome of Atractylodes macrocephala Koidz, BZ), and Gancao (the dry root and rhizome of Glycyrrhiza uralensis Fisch., Glycyrrhiza inflata Bat. Or Glycyrrhiza glabra L., GC). Modern pharmacological studies have revealed that LGZG has a variety of biological and pharmacological properties, such as the regulation of cardiocerebrovascular function [1], regulation of lipid metabolism and blood circulation [2], and anti-inflammation effects [3]. At present, LGZG has been widely used in clinics for the prevention and treatment of heart failure [4], acute 
myocardial infarction [5], nonalcoholic fatty liver [6], obesity [7], hyperlipidemia [8], gastrointestinal function regulation [9], and many other diseases [10, 11]. However, combined with the present stage of research, most of the studies on LGZG have been restricted to the main research index of licorice-related components, while the related components in other herbal medicines are rarely involved, or the overall quality evaluation indexes are not comprehensive, or the range of mass error is excessive, which results in a limited accuracy $[12,13]$. Considering that licorice is only used as a guide medicine in the prescription, the comprehensiveness and objectivity of the method cannot be guaranteed.

As we all know, compound prescriptions of TCM are related to many different herbal medicines, which contain a variety of chemical components and more complex interactions. Generally speaking, after oral administration, the chemical components of the drugs can produce specific synergism or antagonism through blood circulation in multiple organs and targets of the whole body. Its prototype components or metabolites play a corresponding therapeutic role through specific pathways, such as participating in the metabolic regulation, or regulating the homeostasis of microorganisms, and finally causing the overall level of metabolites to change to the normal level. Therefore, it is particularly necessary to establish a comprehensive and systematic research for the chemical constituents of LGZG.

Thus, our study established a quality evaluation system for LGZG using both UPLC-MS/MS and network pharmacology method, which has important guiding significance to help us clarify the pharmacodynamic material basis and its mechanism.

\section{Materials and Methods}

2.1. Materials and Reagents. The abovementioned Chinese herbal medicines were purchased from the following places of origin: FL (batch number: YF18081902, source: Yunnan China; batch number: YF18081914, source: Anhui China; batch number: FL180315, source: Shanxi China), GZ (batch number: YF18083004, source: Guangxi-Botang China; batch number: GZ180321, source: Guangdong China; batch number: 1710203, source: Guangxi-Guilin China), BZ (batch number: YF18070702, source: Zhejiang China; batch number: YF18070603, source: Hebei China; batch number: YF18070802, source: Anhui China), and GC(batch number: YF18063004, source: Neimeng China; batch number: YF18062905, source: Xinjiang China; batch number: YF18062907, source: Gansu China) were provided by Jinnuokang Co., Ltd. (Shijiazhuang, China), which followed identification by Professor Baohui Sun (Hebei Provincial Drug Inspection Institute, Shijiazhuang, China). The voucher specimens have been deposited in the herbarium of the Hebei University of Chinese Medicine.

The purities of all reference standards were higher than 93\%, containing cinnamic acid, cinnamaldehyde, atractylenolide I, atractylenolide II, atractylenolide III, liquiritin, glycyrrhetinic acid, caffeic acid, chlorogenic acid, neochlorogenic acid, and cryptochlorogenic acid attained from the National Institutes for Food and Drug Control (Beijing, China); poricoic acid $\mathrm{A}$, poricoic acid $\mathrm{B}$, polyporenic acid $\mathrm{C}$, isochlorogenic acid $\mathrm{A}$, isochlorogenic acid $\mathrm{B}$, and isochlorogenic acid $\mathrm{C}$ were purchased from the Chengdu Desite Bio-Technology Co., Ltd. (Chengdu, China); isoliquiritin apioside and coumarin were obtained from Shanghai Yuanye Bio-Technology Co., Ltd. (Shanghai, China); isoliquiritin, dehydrotumulosic acid, pachymic acid, 4-hydroxybenzoic acid, and neoisoliquiritin were purchased from Chengdu Pusi Bio-Technology Co., Ltd. (Chengdu, China); glycyrrhizic acid, liquiritigenin, and rutin were obtained from Chengdu Mansite Bio-Technology Co., Ltd. (Chengdu, China); and licochalcone A was purchased from Chengdu Pufei De Bio-Technology Co., Ltd. (Chengdu, China).

Methanol of the HPLC grade was obtained from Fisher Scientific (Fair Lawn, NJ, USA). Acetonitrile of the HPLC grade, together with formic acid of the LC-MS grade, was purchased from Merck (Darmstadt, Germany). Purified water was purchased from Watsons Corporation (Guangzhou, China). The vacuum freeze-dryer was purchased from Beijing Boyikang Experimental Instrument (Beijing, China). All other chemicals and reagents used were of HPLC grade.

\subsection{Standards and Sample Preparation}

2.2.1. Sample Preparation. Following the ancient prescription, herbal medicines, including FL $55.2 \mathrm{~g}, \mathrm{GZ} 41.4 \mathrm{~g}, \mathrm{BZ}$ $41.4 \mathrm{~g}$, and GC $27.6 \mathrm{~g}$, were taken together with $1200 \mathrm{~mL}$ purified water. Until the extracted solution remained $600 \mathrm{~mL}$, it was filtered through a 200-mesh sieve and freezedried. For details of the origin of 18 batches of sample medicinal materials, see Table 1

The sample of LGZG powder(1.0 g) was accurately weighed, dissolved in $70 \%$ methanol-water $(25 \mathrm{~mL})$, and subjected to ultrasonic extraction for $15 \mathrm{~min}$. The solution was weighed again and after the loss in weight was made up with methanol, the obtained solution was then subjected to centrifugation at $4000 \mathrm{rpm}$ for $10 \mathrm{~min}$, and the supernatant was filtered through a $0.22 \mu \mathrm{m}$ nylon membrane (Millipore, USA) prior to analysis. All solutions are stored at $4^{\circ} \mathrm{C}$ before use.

2.2.2. Preparation of Reference Solutions. The reference substance, with the precise weight, was dissolved in HPLC Grade methanol separately. A final mixed standard solution was prepared from all the solutions with the concentrations of poricoic acid A $0.02100 \mu \mathrm{g} / \mathrm{mL}$, poricoic acid B $0.02150 \mu \mathrm{g} /$ $\mathrm{mL}$, glycyrrhizic acid $28.29 \mu \mathrm{g} / \mathrm{mL}$, glycyrrhetinic acid $0.03168 \mu \mathrm{g} / \mathrm{mL}$, liquiritin $32.64 \mu \mathrm{g} / \mathrm{mL}$, isoliquiritin $4.212 \mu \mathrm{g} /$ $\mathrm{mL}$, liquiritigenin $12.33 \mu \mathrm{g} / \mathrm{mL}$, isoliquiritin apioside $5.362 \mu \mathrm{g} / \mathrm{mL}$, cinnamic acid $7.096 \mu \mathrm{g} / \mathrm{mL}$, caffeic acid $0.1697 \mu \mathrm{g} / \mathrm{mL}$, neochlorogenic acid $1.655 \mu \mathrm{g} / \mathrm{mL}$, chlorogenic acid $1.615 \mu \mathrm{g} / \mathrm{mL}$, cryptochlorogenic acid $0.5528 \mu \mathrm{g} / \mathrm{mL}$, isochlorogenic acid A $0.3894 \mu \mathrm{g} / \mathrm{mL}$, isochlorogenic acid B $0.2430 \mu \mathrm{g} / \mathrm{mL}$, isochlorogenic acid C $0.3353 \mu \mathrm{g} / \mathrm{mL}$, atractylenolide I $0.6000 \mu \mathrm{g} / \mathrm{mL}$, atractylenolide II $0.3300 \mu \mathrm{g} / \mathrm{mL}$, 
TABLE 1: Origin of 18 batch samples of TCM.

\begin{tabular}{lcccc}
\hline Batches & FL & GZ & BZ & GZ \\
\hline $\mathbf{1}$ & YF18081902 & YF18083004 & YF18070702 & YF18063004 \\
$\mathbf{2}$ & YF18081902 & YF18083004 & YF18070702 & YF18063004 \\
$\mathbf{3}$ & YF18081902 & YF18083004 & YF18070702 & YF18063004 \\
$\mathbf{4}$ & YF18081902 & YF18083004 & YF18070702 & YF18063004 \\
$\mathbf{5}$ & YF18081902 & YF18083004 & YF18070702 & YF18062905 \\
$\mathbf{6}$ & YF18081902 & YF18083004 & YF18070702 & YF18062905 \\
$\mathbf{7}$ & YF18081914 & GZ180321 & YF18070603 & YF18062905 \\
$\mathbf{8}$ & YF18081914 & GZ180321 & YF18070603 & YF18062907 \\
$\mathbf{9}$ & YF18081914 & GZ180321 & YF18070603 & YF18062907 \\
$\mathbf{1 0}$ & FL180315 & 1710203 & YF18070802 & YF18062907 \\
$\mathbf{1 1}$ & FL180315 & 1710203 & YF18070802 & YF18070802 \\
$\mathbf{1 2}$ & FL180315 & 1710203 & YF18070702 & YF18063004 \\
$\mathbf{1 3}$ & YF18081902 & GZ180321 & YF18070702 & YF18063004 \\
$\mathbf{1 4}$ & YF18081902 & GZ180321 & YF18070802 & YF18062905 \\
$\mathbf{1 5}$ & YF18081914 & 1710203 & YF18070802 & \\
$\mathbf{1 6}$ & YF18081914 & 1710203 & YF18070603 & \\
$\mathbf{1 7}$ & FL180315 & YF18083004 & & \\
\hline
\end{tabular}

atractylenolide III $0.9400 \mu \mathrm{g} / \mathrm{mL}$ and coumarin $2.736 \mu \mathrm{g} / \mathrm{mL}$. All the standard solutions were stored at $4{ }^{\circ} \mathrm{C}$ and filtered through a $0.22 \mu \mathrm{m}$ nylon membranes (Millipore, USA) before analysis.

\subsection{Chromatographic and Mass Spectral Conditions}

2.3.1. UPLC-Q-TOF-MS/MS Analysis. UPLC-Q-TOFMS/MS analysis was completed with an Acquity TM UPLC system (Waters, Milford, MA, USA), which was equipped with a quaternary solvent manager, an automatic sample manager-FTN, and a PDA e $\lambda$ detector. The samples were separated on a Waters ACQUITY UPLC BEH $\mathrm{C} 18(100 \mathrm{~mm} \times 2.1 \mathrm{~mm}, 1.7 \mu \mathrm{m})$ maintained at $35^{\circ} \mathrm{C}$, using a mixed mobile phase consisted of $0.1 \%$ formic acid in pure water (A) and acetonitrile (B). A gradient elution program was as follows: $0-3 \mathrm{~min}, 5 \% \mathrm{~B} ; 3-20 \mathrm{~min}, 5 \%-30 \% \mathrm{~B}$; 20-25 min, 30\% B; $25-50 \mathrm{~min}, 30 \%-80 \%$ B; $50-55 \mathrm{~min}, 80 \%$ $\mathrm{B}$; and $55.1-60 \mathrm{~min}, 5 \% \mathrm{~B}$. The flow rate and the injection volume were set at $0.3 \mathrm{~mL} / \mathrm{min}$ and $2 \mu \mathrm{L}$, respectively.

The mass analysis was implemented by a Triple TOFTM $6600+(\mathrm{AB}$ SCIEX, Foster City, CA, USA), which is equipped with an electrospray ionization (ESI) source. The MS analysis was performed in both positive ionization mode and negative ionization mode by full scan mode. The optimized parameters were as follows: ion spray voltage (ISV), $5.5 \mathrm{kV}(\mathrm{ESI}+)$ or $-4.5 \mathrm{kV}$ (ESI-); ion source temperature (TEM), $550^{\circ} \mathrm{C}$; ion source gas 1 (GS1), 55 psi; ion source gas 2 (GS2), 55 psi; curtain gas (CUR), 35 psi, scan range of TOF-MS, $\mathrm{m} / z 100-1500 \mathrm{Da}$; and scan range of product ion, $\mathrm{m} / z$ 100-1500 Da. IDA-MS/MS conditions were as follows: accumulation time was $0.05 \mathrm{~s}$; high sensitivity mode was set, excluding isotopes were within $4 \mathrm{Da}$; declustering potential (DP) was $80 \mathrm{~V}$ (ESI+) or $-80 \mathrm{~V}$ (ESI-); collision energy (CE) was $10 \mathrm{eV}$ (MS mode), and 40 and $80 \mathrm{eV}$ (MS2 mode); and collision energy spread (CES) was $20 \mathrm{eV}$. Moreover, in order to ensure accurate mass measurements during the MS experiments, the instrument performed mass accuracy calibration through the CDS system before each experiment. During the experiment, the mass accuracy was calibrated for every three samples. The data acquisition and processing will be analyzed by SCIEX OS-Q 2.0 Software (AB SCIEX, Foster City, CA, USA) and Peak View ${ }^{\circledR} 2.2$ Software (AB SCIEX, Foster City, CA, USA).

2.3.2. UPLC-QQQ-MS/MS Analysis. UPLC-QQQ-MS/MS analysis was performed on the LC-30A UPLC system(Shimadzu, Kyoto, Japan), including DGU-30A3 type online vacuum degasser, LC-30AD-type binary pump, SIL30AC-type automatic sampler, and CTO-30A-type column incubator. The experimental conditions were as follows: Shimpack GIST $\mathrm{C} 18(100 \mathrm{~mm} \times 2.1 \mathrm{~mm}, 2 \mu \mathrm{m})$ chromatographic column; column temperature, $35^{\circ} \mathrm{C}$; flow rate, $0.3 \mathrm{~mL} / \mathrm{min}$; and injection volume, $2 \mu \mathrm{L}$. The mobile phase was selected to be water containing $0.1 \%$ formic acid (A) and acetonitrile (B) with the following gradient elution programme: $0-17 \mathrm{~min}$, 5-17\% B; $17-31 \mathrm{~min}, 17-75 \% \mathrm{~A} ; 31-32 \mathrm{~min}, 75 \% \mathrm{~A}$; and 32.1-35 $\mathrm{min}, 5 \% \mathrm{~A}$. In terms of mass spectrometry, we chose QTRAP 4500 (AB SCIEX, Foster City, CA, USA) coupled with an electrospray ionization (ESI) source. The analytical method adopts the MRM mode, with the positive and negative ion switching detection method. The ion spray voltage (ISV) was $5.5 \mathrm{kV}$ (ESI+) or $-4.5 \mathrm{kV}$ (ESI-); ion source temperature, (TEM) $550^{\circ} \mathrm{C}$; ion source gas 1 (GS1), 55 psi; ion source gas 2 (GS2), 55 psi; and curtain gas (CUR), 35 psi.

\subsection{Validation of the Quantitative Method}

2.4.1. Calibration Curve, LOD, and LOQ. The mixed reference substance solution was diluted step by step to form a mixed reference substance solution with six concentration gradients. According to the condition of item 2.3.2, the extracted ion current chromatogram of each reference substance was obtained. With the concentration of each reference substance $(\mathrm{X})$ and peak area $(\mathrm{Y})$, the standard 
curves were drawn and linear regressions were carried out. The mixed reference solution was diluted step by step and determined until the quantification limit (LOQ) and detection limit (LOD) were determined when the signal-tonoise ratio $(\mathrm{S} / \mathrm{N})$ was 10 and 3 , respectively.

2.4.2. Precision, Repeatability, Stability, and Recovery. In order to investigate the precision, the mixed reference solution and sample solution were injected continuously for 6 times, the peak areas were recorded, and the RSD values were calculated. In the repetitive investigation, 6 samples of the same batch were taken and prepared in parallel. Through the sequential injection analysis of the samples, the peak area and the RSD value were calculated. According to the stability test, the solution of the same batch of samples was injected and analyzed at $0,4,8,12,18$, 24,36 , and $48 \mathrm{~h}$, respectively. The recovery rates were investigated by adding high, medium, and low levels of reference solution $(150,100$, and $50 \%$ of the known amount, respectively) to the same batch of samples, with 3 parallel samples at each level. Recovery $(\%)=($ detected value - original value)/added amount $\times 100 \%$.

2.5. Strategy. Through searching and sorting out the previous literature, the chemical composition information database of four kinds of TCM in LGZG was established, including compound name, molecular formula, exact molecular weight, structural formula, and other essential information. For compounds with reference substances, it was confirmed by comparing the chromatographic retention time and mass spectrometry data. For unknown compounds, the OS software was used to compare the self-built composition database, TCM MS/MS database (supplied by AB SCIEX) and online Chemspider database. The screening conditions were that the quality deviation was less than $1 \mathrm{ppm}$ (FL less than $3 \mathrm{ppm}$ ). The fragmentation pattern of representative components was analyzed by the MS/MS spectrogram of the compound.

According to the results of chemical composition research, a network pharmacology research was carried out to screen biomarkers, which were taken as the references to carry out the quality evaluation research. On this basis, the study established a quantitative study with high accuracy and good stability. Combined with the method of statistical analysis, the differences between different areas of herbal medicines were analyzed, which will provide a theoretical basis for the quality evaluation of TCM.

\section{Results and Discussion}

3.1. Identification of the Chemical Components in LGZG by UPLC-ESI-Q-TOF-MS. By introducing the UPLC-ESI-QTOF-MS analysis method based on DBS-IDA data acquisition technology, the spectrum signals were 45,205 and 43,188 measured in positive mode and negative ion detection mode, respectively. Combined with reference substance comparison, literature research, and MS/MS information, a total of 149 chemical constituents in LGZG were identified, including 63 of flavonoids, 30 of triterpenes, 22 of phenylpropanoids, 13 of organic acids, 6 of lactones, 5 of alkaloids, 4 of anthraquinones, and 6 of other compounds. The typical total ion chromatograms of positive and negative ion modes are shown in Figure 1. All the compounds and related information are shown in Tab.S1.

3.1.1. Identification of Flavonoids. As the most abundant compounds in nature, flavonoids are not only the main chemical components of many kinds of TCM, but also the most important therapeutic active components. In this study, a total of 63 flavonoids were identified or preliminarily inferred, mainly from GZ and GC. These components can be further divided into flavonoid aglycones, O-glycosyl flavonoids, and C-glycosyl flavonoids.

(1) Flavonoid Aglycones. The main cracking mode of flavonoids is the Retro-Diels-Alde (RDA) fragmentation mechanism, which can also eliminate small neutral molecular fragments, such as $\mathrm{CH}_{3}(\mathrm{~m} / z$ 15.0240 Da), O (m/z 15.9949 Da), $\mathrm{H}_{2} \mathrm{O}\left(\mathrm{m} / z\right.$ 18.0106 Da), $\mathrm{CO}\left(\mathrm{m} / z\right.$ 27.9949 Da), $\mathrm{CO}_{2}(\mathrm{~m} / z$ $43.9898 \mathrm{Da})$, and other substituents on benzene ring [14-16]. Take isoflavones Eurycarpin $\mathrm{A}\left(\mathrm{C}_{20} \mathrm{H}_{18} \mathrm{O}_{5},[\mathrm{M}-\mathrm{H}]^{-}, \mathrm{P} 103\right)$ as an example, which is observed at $\mathrm{m} / z$ 337.1090. Firstly, the fragment ion $\mathrm{m} / z 293.0462$ is produced by losing $\mathrm{CO}_{2}$. Meanwhile, the molecule can remove the isopentenyl group $\left(\mathrm{C}_{5} \mathrm{H}_{9}\right)$ from the benzene ring to form the $\mathrm{m} / z 268.0377$ fragment ion $(\mathrm{m} / z-69)$, which can continue to remove $-\mathrm{CO}_{2}$ to form $\mathrm{m} / z 224.0480$ fragment ion. Finally, on the basis of 368 fragments, $\mathrm{m} / z 135.0091$ and $\mathrm{m} / z 117.0346$ fragments are produced through the RDA fragmentation mechanism. The fragmentation pathway of Eurycarpin A is shown in Figure 2.

As a branch of flavonoids, isoflavanes do not contain $\mathrm{CO}$ in the $\mathrm{C}_{3}$ ring, which cannot form a conjugated system.

This leads to its unstable structure and easy fracture at the $\mathrm{C}_{3}$ ring. We explain the fragmentation mechanism of isoflavane through compounds $7,4^{\prime}$-dihydroxy- $3^{\prime}$-methoxyisoflavan $\left(\mathrm{C}_{16} \mathrm{H}_{16} \mathrm{O}_{4},[\mathrm{M}-\mathrm{H}]^{-}, \mathrm{P} 87\right)$. The component exhibited a parent ion at $\mathrm{m} / z$ 271.1000, which yielded daughter ions at $\mathrm{m} / z 256.0719$ and 241.0512 by losing a $\mathrm{CH}_{3}$ and $\mathrm{CH}_{2} \mathrm{O}$ group, respectively. Besides, components were cleaved by RDA reaction to form $\mathrm{m} / z \quad 121.0296$ and 149.0600. The most important thing is that the $\mathrm{C}-\mathrm{C}$ and $\mathrm{C}-\mathrm{O}$ bonds at $\mathrm{C}_{3}$ can be broken, so that the molecule is divided into two new fragment ions $\mathrm{m} / z 109.0305$ and 135.0447. The fragmentation pathway of $7,4^{\prime}$-dihydroxy- $3^{\prime}$ methoxyisoflavan was shown in Figure 3.

According to the rules mentioned above, a total of 46 flavonoids were identified or inferred, including 13 isoflavones (labeled as P50, 84, 85, 103, 110, 115, 119, 120, 123, $132,135,140,141), 8$ dihydroflavonoids(labeled as P41, 67, $81,100,101,118,125,131), 8$ chalcone (labeled as P58, 72, 73, 74, 83, 97, 109, 112), 5 flavonoids(labeled as P49, 60, 65, 114, 124), 3 isoflavones(labeled as P87, 99, 126), 2 flavonols (labeled as P51, 76), 2 dihydroflavonols(labeled as P21, 39), 2 procyanidins(labeled as P20, 28), 1 xanthones(labeled as P47), 1 isoflavene (labeled as P93), and 1 2-(2-phenylethyl) chromone(labeled as P69). 


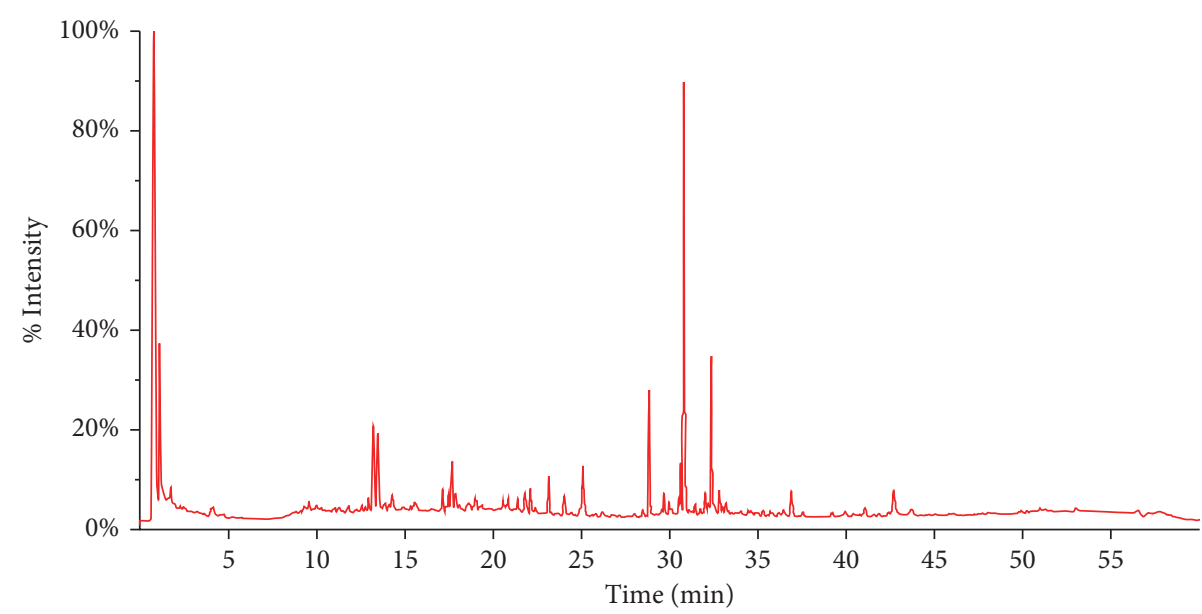

(a)

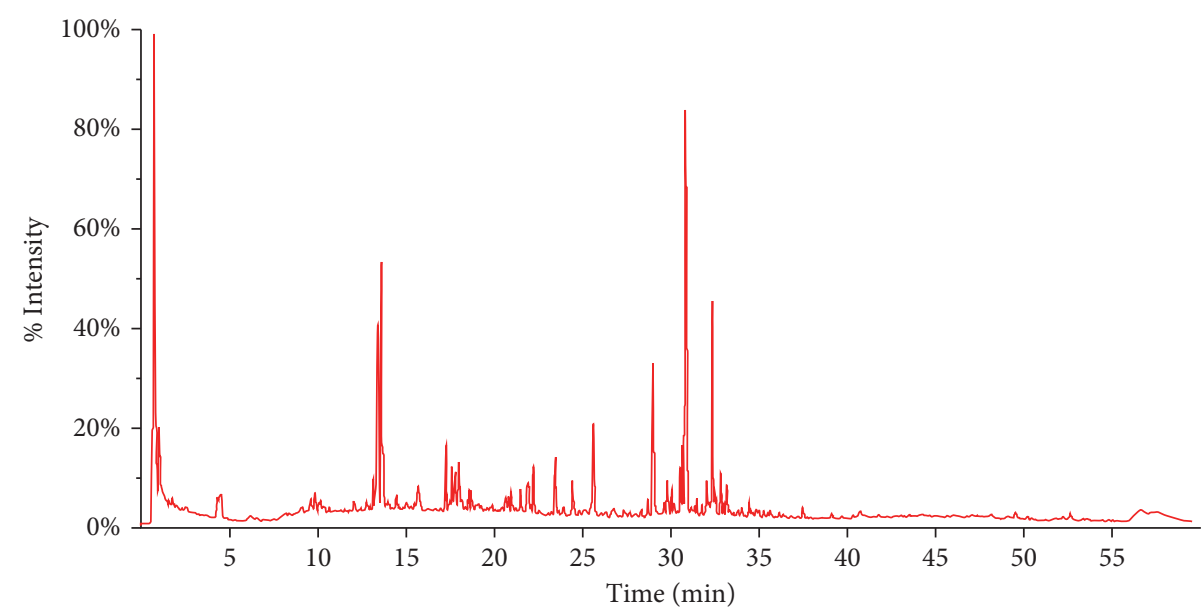

(b)

FIGURE 1: The total ion chromatograms of LGZC (a) under the positive ion mode and (b) under the negative ion mode.

(2) O-Glycosyl Flavonoids. Natural flavonoids mainly exist in plants in the form of glycosides combined with glycosyl groups (monosaccharides, disaccharides, trisaccharides, polysaccharides, etc.). According to the glycoside bond atoms connected to the glycosyl groups, natural flavonoids are divided into O-glycosyl flavonoids and C-glycosyl flavonoids.

For O-flavonoid glycosides, the glycosyl connection position is related to the parent nuclear structure of flavonoids. In terms of flavonoids, dihydroflavonoids and isoflavones, most of them form monosaccharides on 7-OH. While flavonols and dihydroflavonol glycosides form monosaccharides on 3-OH, 7$\mathrm{OH}, 3^{\prime}-\mathrm{OH}$ and $4^{\prime}-\mathrm{OH}$, or disaccharides on $3-\mathrm{OH}$ and $7-\mathrm{OH}, 3-$ $\mathrm{OH}$ and $4^{\prime}-\mathrm{OH}$ or $7-\mathrm{OH}$ and $4^{\prime}-\mathrm{OH}$.

Dihydroflavone $\mathrm{O}$-glycoside liquiritin apioside $\left(\mathrm{C}_{26} \mathrm{H}_{30} \mathrm{O}_{13},[\mathrm{M}-\mathrm{H}]^{-}, \mathrm{P} 45\right)$ and chalcone O-glycoside isosalipurposide $\left(\mathrm{C}_{21} \mathrm{H}_{22} \mathrm{O}_{10},[\mathrm{M}-\mathrm{H}]^{-}, \mathrm{P} 59\right)$ are used as standards for explanation. The two compounds are first removed from the glycosyl group: liquiritin apioside removes two molecules of glycosyl $\left(\mathrm{C}_{5} \mathrm{H}_{8} \mathrm{O}_{4}, \mathrm{~m} / z 132.0423\right.$; $\mathrm{C}_{6} \mathrm{H}_{10} \mathrm{O}_{5}, \mathrm{~m} / z$ 162.0528); and isosalipurposide loses a molecule $\left(\mathrm{C}_{6} \mathrm{H}_{10} \mathrm{O}_{5}\right)$. On this basis, new fragment ions are further formed by RDA cleavage (liquiritin apioside: $\mathrm{m} / z$ 135.0106, 119.0517; isosalipurposide: $\mathrm{m} / z$ 151.0048, 119.0511, Figure 4).

(3) C-Glycosyl Flavonoids. In C-glycosides flavonoids, the glycosyl groups are mostly linked at the position of $\mathrm{C}_{6}$ or $\mathrm{C}_{8}$, or at both $\mathrm{C}_{6}$ and $\mathrm{C}_{8}$. Because the $\mathrm{C}-\mathrm{C}$ bond of flavonoid $\mathrm{C}$-glycosides is unstable, the ring-opening reaction of glycosyl groups mainly occurs in the process of cleavage. The continuous neutral loss of $\mathrm{H}_{2} \mathrm{O}(\mathrm{m} / z 18.0106 \mathrm{Da})$ is dominant in the positive ion mode, and the glycosyl neutral loss is mainly in the negative ion mode, such as hexose neutral loss $\left(\mathrm{C}_{4} \mathrm{H}_{8} \mathrm{O}_{4} \mathrm{~m} / z 120.0423\right.$ or $\mathrm{C}_{3} \mathrm{H}_{6} \mathrm{O}_{3} \mathrm{~m} / z$ 90.0317) and pentose neutral loss $\left(\mathrm{C}_{3} \mathrm{H}_{6} \mathrm{O}_{3} \mathrm{~m} / z 90.0317\right.$ or $\mathrm{C}_{2} \mathrm{H}_{4} \mathrm{O}_{2} \mathrm{~m} / z$ 60.0211] [17]. Schaftoside $\left(\mathrm{C}_{26} \mathrm{H}_{28} \mathrm{O}_{14},[\mathrm{M}-\mathrm{H}]^{-}, \mathrm{P} 30\right)$ is used as a reference to explain its cracking law. According to the information provided by the mass spectrogram, the parent ion of schaftoside is $\mathrm{m} / z 563.1431$ and a series of characteristic ions are $\mathrm{m} / z$ 503.1198, 473.1102, 443.0990, 383.0781, and 353.0678 , whose mass difference of each fragment ion is an integral multiple of $\mathrm{CH}_{2} \mathrm{O}(\mathrm{m} / z$ 30.0106). The specific cracking process is shown in Figure 5. On the basis of the above rules, 17 flavonoid glycosides were identified, 


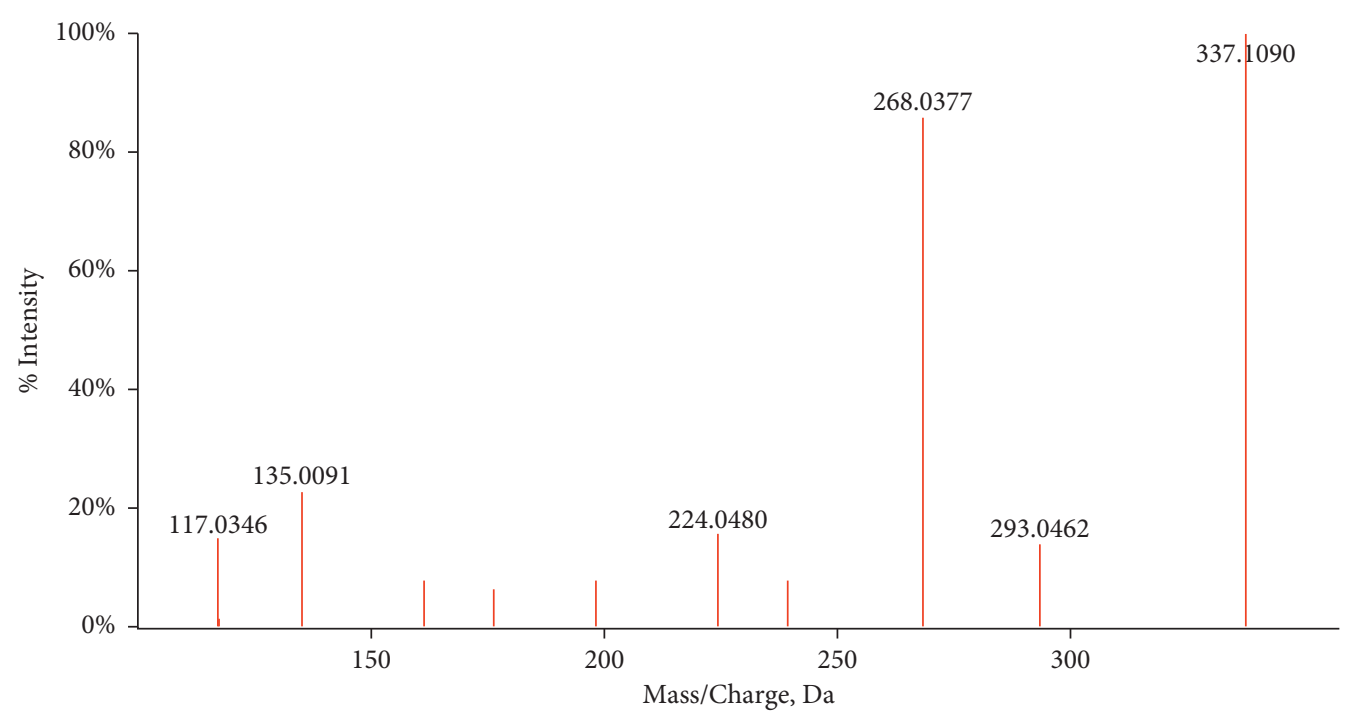

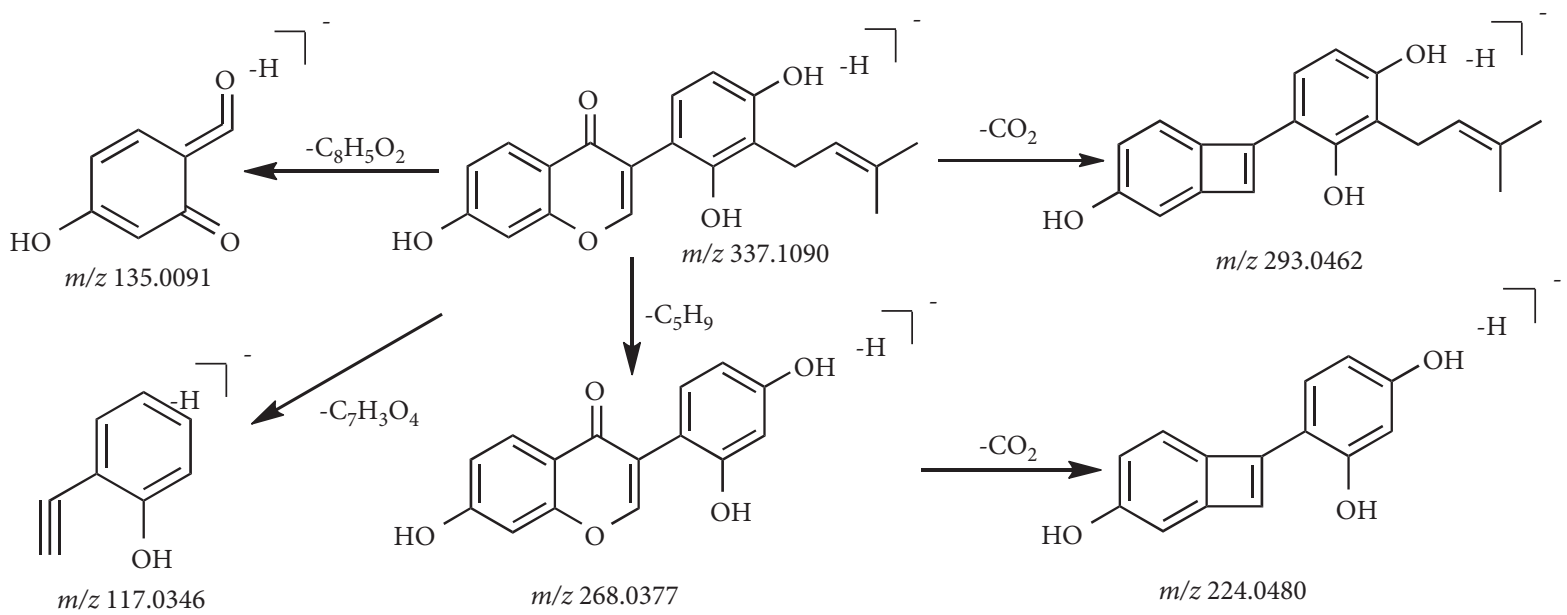

FIgURE 2: The fragmentation pathway of eurycarpin A.

including $13 \mathrm{O}$-glycosyl flavonoids(labeled as P34, 35, 37, 38, $40,43,45,55,59,66,68,70,71)$ and 4 flavonoid C-glycosyl flavonoids(labeled as P24, 30, 44, 53).

3.1.2. Identification of Triterpenoids. The basic skeleton of triterpenoids is connected by 6 isoprene structural units. Considering the skeleton with multiple six-membered rings in the structure is extremely stable and not easy to break bonds, higher collision energy is needed in the process of mass spectrometry pyrolysis. Triterpenes and their glycosides are easy to remove sugar groups (such as glucose) and ring-linked substituents, including $\mathrm{OH}$ (lost in the form of $\left.\mathrm{H}_{2} \mathrm{O}, \mathrm{m} / z 18.0106 \mathrm{Da}\right), \mathrm{COOH}$ (lost in the form of $\mathrm{CO}_{2}, \mathrm{~m} / z$ $43.9898 \mathrm{Da})$, and other substituent groups [18].

For our study, most of triterpenoids in the prescription mainly come from FL and GC. With reference to tumulosic acid $\left(\mathrm{C}_{31} \mathrm{H}_{50} \mathrm{O}_{4},[\mathrm{M}-\mathrm{H}]^{-}, \mathrm{P} 130\right)$, the difference between parent ion and daughter ion is $\mathrm{m} / z$ 62.0004. That is to say, the compound loses $\mathrm{CO}_{2}$ and $\mathrm{H}_{2} \mathrm{O}$ at one time (parent iondaughter ion: $\mathrm{m} / z$ 485.3639-423.3196). The following is to compare the differences of fragmentation mechanism between the positive and negative ion mode with pachymic acid $\left(\mathrm{C}_{33} \mathrm{H}_{52} \mathrm{O}_{5}, \mathrm{P} 146\right)$. In the positive ion mode, when the collision energy is set to 40 , the parent ion peak is not displayed, and the daughter ion is $\mathrm{m} / z$ 511.3770, 451.3562, 355.2625, and 295.2422. Among them, $\mathrm{m} / z 511.3770$ is formed by removing $\mathrm{H}_{2} \mathrm{O}$ on the basis of the parent ion, and the subsequent daughter ions lose $\mathrm{CH}_{3} \mathrm{COOH}$, branchedchain $\mathrm{C}_{7} \mathrm{H}_{12}$, and $\mathrm{C}_{2} \mathrm{H}_{4} \mathrm{O}_{2}$. However, in negative ion mode, when the collision energy is set to 40 , basically only 527 single peak can be displayed (there is one peak at 465 , and the intensity is only $2.37 \%$ ). Increasing the collision energy to 80 , the parent ion is at $\mathrm{m} / z 527.3737$ and the daughter ion peaks are at $\mathrm{m} / z 467.3529,465.3368,405.3179$, and 293.1899 in sequence. On the basis of the parent ion, on the one hand, it can lose $\mathrm{CO}_{2}$ and $\mathrm{H}_{2} \mathrm{O}$ to form the $\mathrm{m} / z 465.3368$ ion. On the other hand, it can also lose the $\mathrm{CH}_{3} \mathrm{COOH}$ to form the $\mathrm{m} / z 467.3529$ ion, which can continue to complete the loss of another group of ions, forming a 405.3179 peak. Finally, the branched-chain $\mathrm{C}_{8} \mathrm{H}_{16}$ was removed again to form the 293.1899 peak. The detailed cracking process is shown in Figure 6. According to the rules discussed above, a total of 30 compounds are inferred (labeled as P82, 86, 88, 89, 90, 91, 92, $102,105,106,107,108,111,121,128,129,130,133,134,136$, $137,138,139,142,143,144,145,146,148,149)$. 

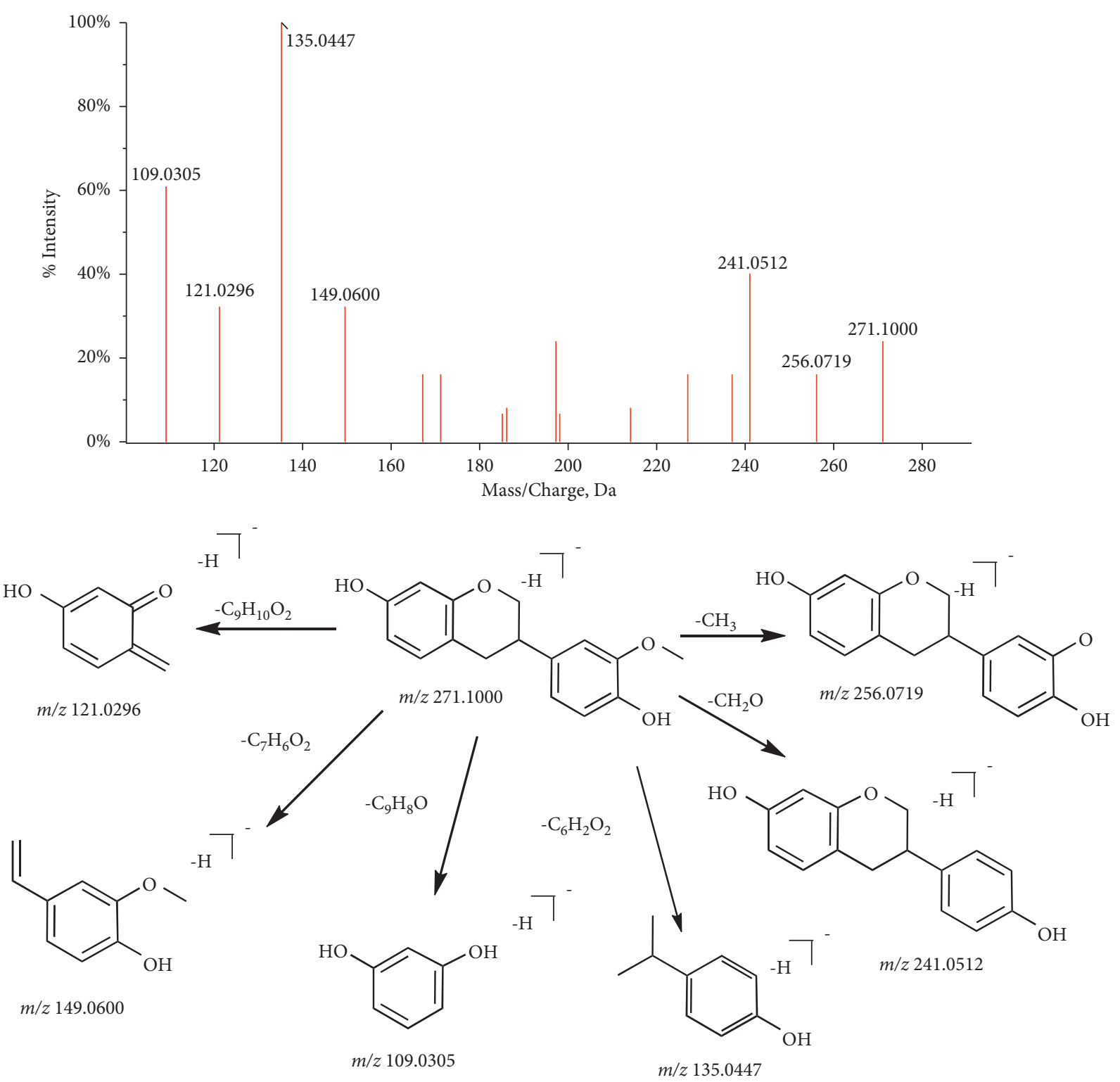

Figure 3: The fragmentation pathway of 7,4'-dihydroxy-3'-methoxyisoflavan.

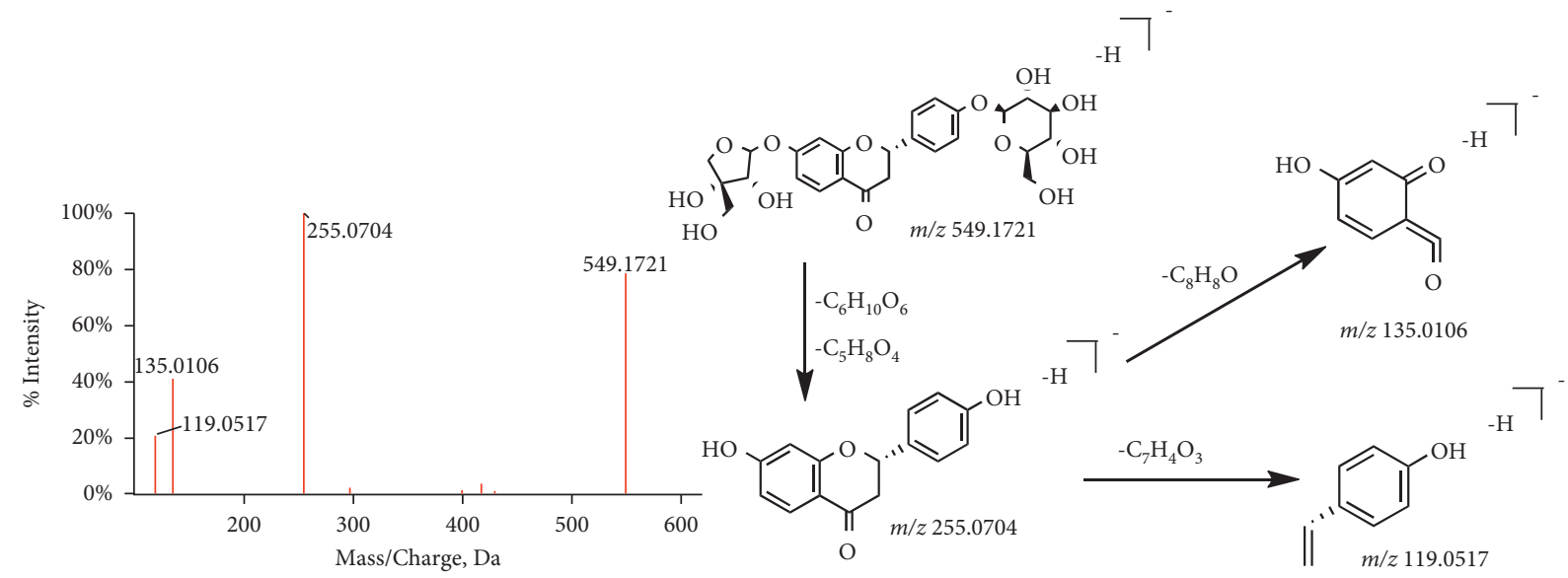

(a)

Figure 4: Continued. 

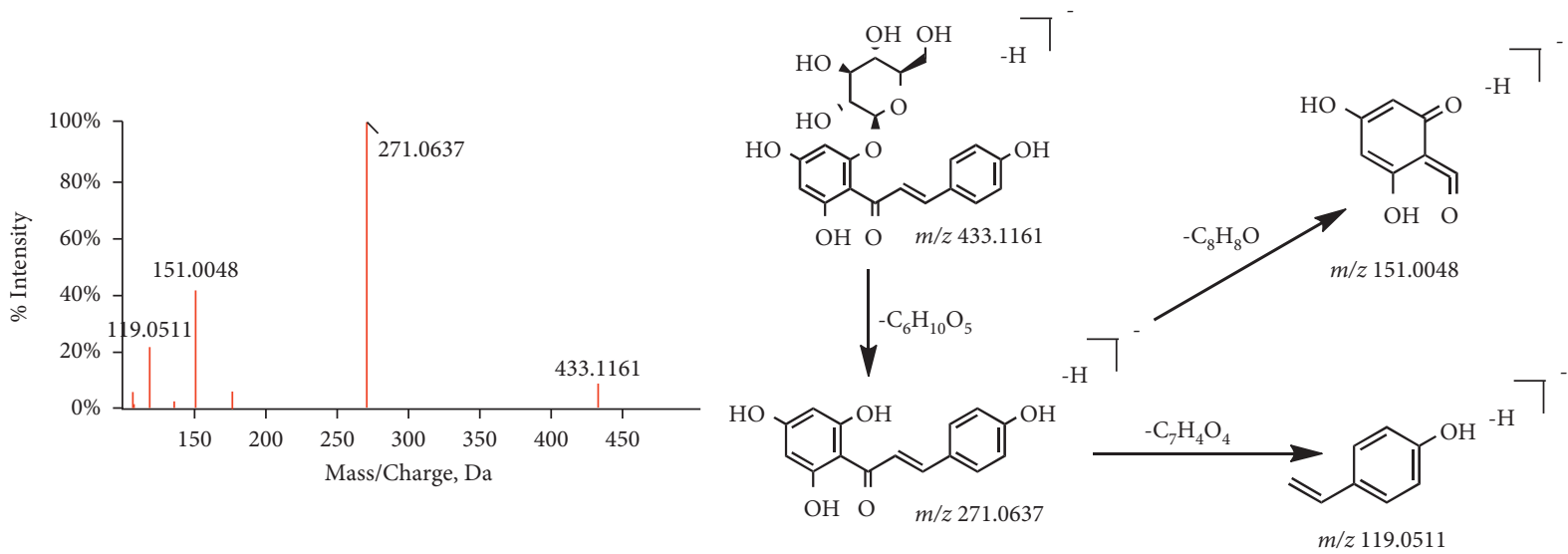

(b)

Figure 4: The fragmentation pathway of O-glycosyl flavonoid. The fragmentation pathway of (a) liquiritin apioside and (b) isosalipurposide.
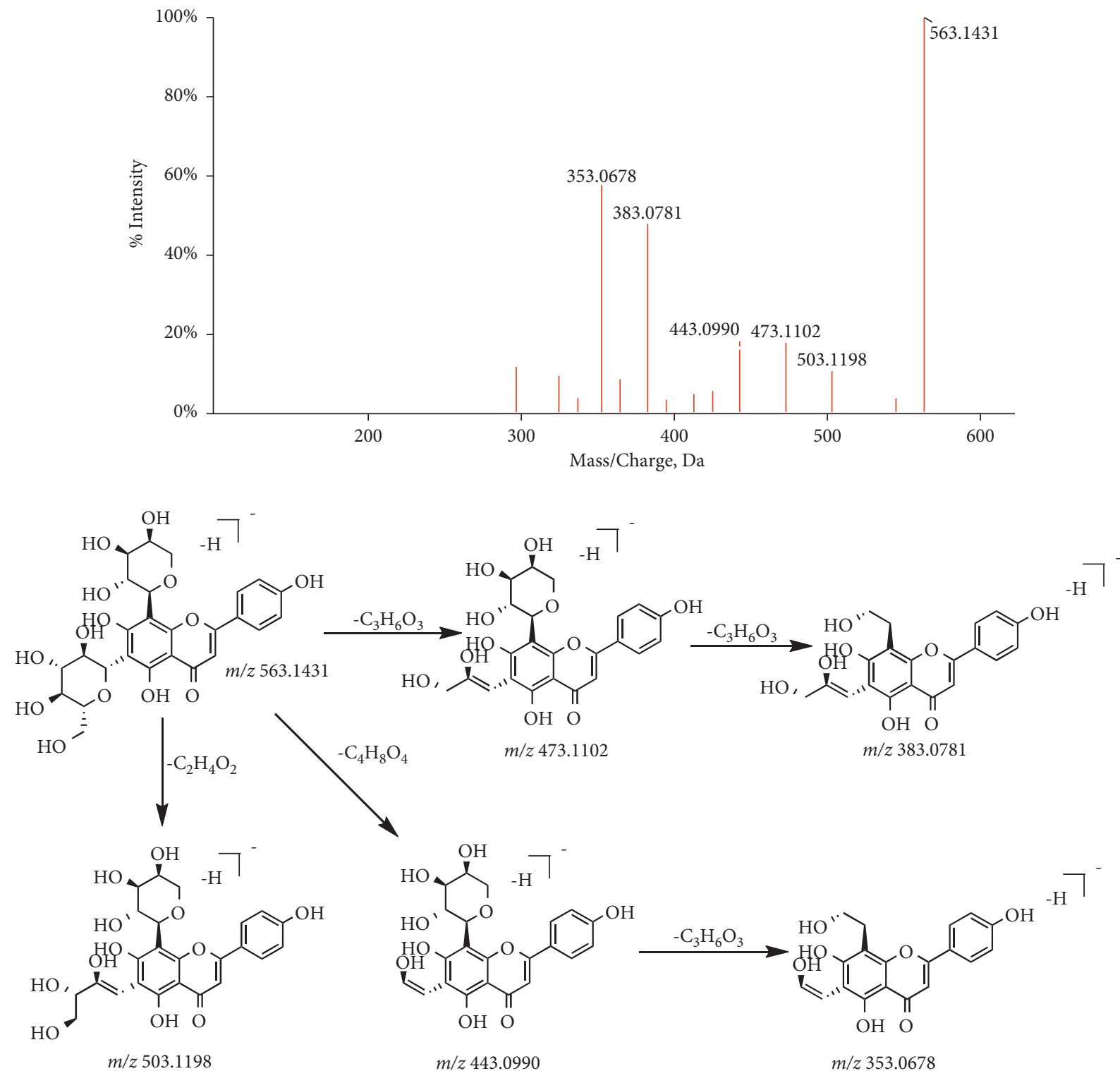

FIGURE 5: The fragmentation pathway of schaftoside. 


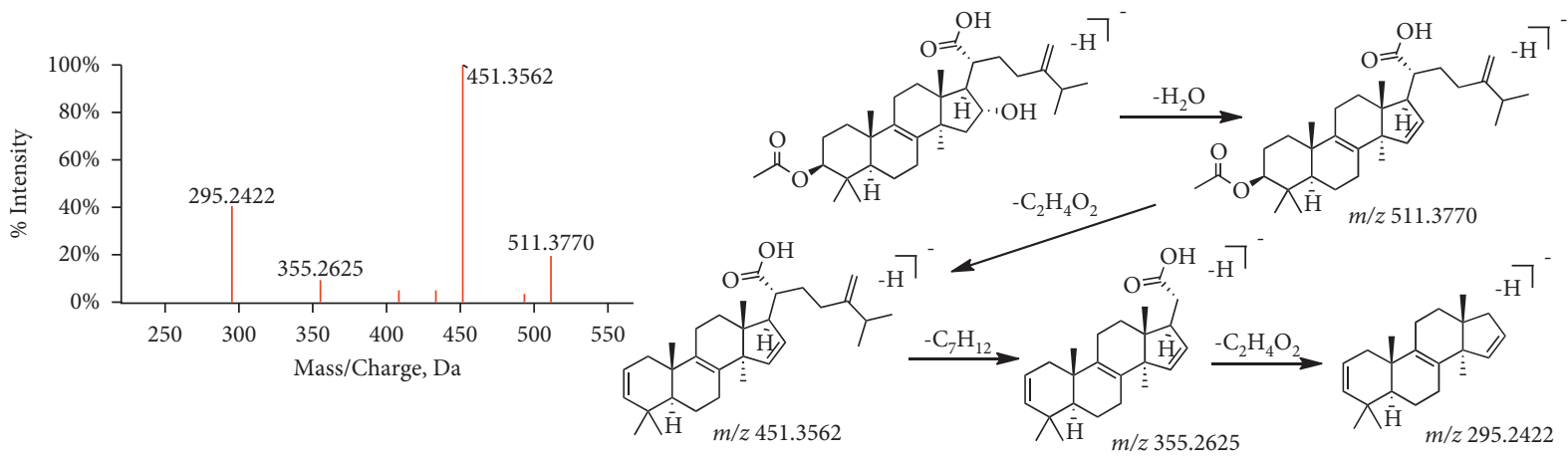

(a)

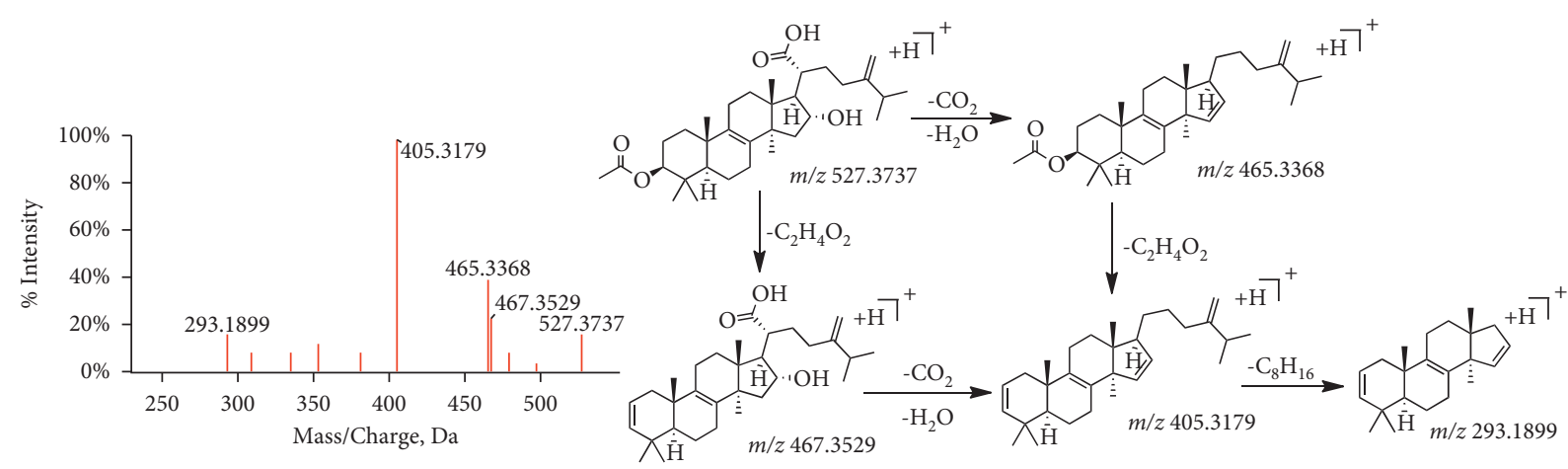

(b)

Figure 6: The fragmentation pathway of pachymic acid. The fragmentation pathway of (a) positive ion mode and (b) negative ion mode.

3.1.3. Identification of Phenylpropanoids. Phenylpropanoid compounds mainly include lignans, coumarins, phenylpropanoids, and their glycosides, which have different structural characteristics. Phenylpropanols are a kind of naturally occurring compound composed of benzene ring and three straight-chain carbon groups $\left(\mathrm{C}_{6}-\mathrm{C}_{3}\right.$ groups). It generally has a phenol structure and is also a phenolic substance. Because caffeoyl groups often exist in its structure, the characteristic fragment ions of $\left[\mathrm{M}-\mathrm{H}-\mathrm{C}_{9} \mathrm{H}_{6} \mathrm{O}_{3}\right]$ can often be obtained in the fragmentation of mass spectrometry. In addition, it is easy to lose $\mathrm{CH}_{3}, \mathrm{H}_{2} \mathrm{O}, \mathrm{CO}, \mathrm{CO}_{2}$, or $\mathrm{COOH}$ to obtain fragment ions with mass loss of $15,18,28,44$, and $45 \mathrm{Da}[19,20]$. For example, compound 2-methoxycinnamic $\operatorname{acid}\left(\mathrm{C}_{10} \mathrm{H}_{10} \mathrm{O}_{3},[\mathrm{M}-\mathrm{H}]^{-}, \mathrm{P} 46\right)$ produced an $\mathrm{m} / z$ peak at 162.0326 upon the elimination of $\mathrm{CH}_{3}$, and this was followed by the loss of $\mathrm{COOH}$ groups in succession to give peaks at $\mathrm{m} / z$ 117.0339(Figure 7(a)).

The basic nuclear structure of coumarin is benzopyranone (cis-2-hydroxycinnamic acid lactone). According to the different substituents and connection modes, coumarin can be divided into simple coumarin, furocoumarins, pyranocoumarins, and other coumarins. Coumarins generally have many $\mathrm{CO}, \mathrm{CO}_{2}, \mathrm{OH}, \mathrm{H}_{2} \mathrm{O}, \mathrm{CH}_{3}$, and $\mathrm{OCH}_{3}$ connected to aromatic rings, so that a series of neutral ion peaks with continuous loss often appear. In addition, coumarins often have common functional groups such as isopentenyl, acetoxy, and 5-carbon unsaturated acyloxy groups, which are also the main characteristics of coumarin compounds [21, 22]. Glycycoumarin displayed the ions at $\mathrm{m} / z 367.1193\left(\mathrm{C}_{21} \mathrm{H}_{19} \mathrm{O}_{6}\right.$,
$\left.[\mathrm{M}-\mathrm{H}]^{-}, \mathrm{P} 104\right)$, with the following diagnostic fragment ions provided by the MS/MS spectrum at $\mathrm{m} / z 337.0719\left(\mathrm{C}_{19} \mathrm{H}_{13} \mathrm{O}_{6}\right.$, $\left.\left[\mathrm{M}-\mathrm{H}-2 \mathrm{CH}_{3}\right]^{-}\right), \quad 309.0414 \quad\left(\mathrm{C}_{18} \mathrm{H}_{13} \mathrm{O}_{5}, \quad\left[\mathrm{M}-\mathrm{H}-2 \mathrm{CH}_{3}-\mathrm{CO}\right]^{-}\right)$, $297.0408 \quad\left(\mathrm{C}_{16} \mathrm{H}_{9} \mathrm{O}_{6}, \quad\left[\mathrm{M}-\mathrm{H}-2 \mathrm{CH}_{3}-\mathrm{C}_{3} \mathrm{H}_{4}\right]^{-}\right), \quad 284.0336$ $\left(\mathrm{C}_{16} \mathrm{H}_{12} \mathrm{O}_{5},\left[\mathrm{M}-\mathrm{H}-2 \mathrm{CH}_{3}-\mathrm{O}-\mathrm{C}_{2} \mathrm{H}\right]^{-}\right)$, and $203.0718\left(\mathrm{C}_{11} \mathrm{H}_{7} \mathrm{O}_{4}\right.$, $\left[\mathrm{M}-\mathrm{H}-\mathrm{C}_{6} \mathrm{H}_{5} \mathrm{O}_{2}-\mathrm{C}_{4} \mathrm{H}_{7}\right]^{-}$) (Figure 7(b)).

Lignans are natural compounds of derivatives formed by the oxidative polymerization of phenylpropanoids, usually in the form of dimers, as well as a few number of trimers and tetramers. Most of them exist in free form in plant wood and resin, and also combine with glycosyl groups to form glycosides. As the phenylpropanoids polymers, the typical characteristic ion fragments of lignans are $\mathrm{M} / 2$ or $\mathrm{M} / 3$ peak, whose response intensity is relatively high. The other cleavage rules are consistent with phenylpropanols [23]. In the mass spectrogram of secoisolariciresinol $\left(\mathrm{C}_{20} \mathrm{H}_{26} \mathrm{O}_{6},[\mathrm{M}-\mathrm{H}]^{-}, \mathrm{P} 64\right)$, it is observed that the parent ion is $\mathrm{m} / z 361.1683$, with the daughter ion $\mathrm{M} / 2-1$ peak $\mathrm{m} / z 179.0712$. The rest of the fragment ions, such as $\mathrm{m} / z$ $165.0559,147.0456$, and 121.0292, are formed by losing $\mathrm{CH}_{3}$, $\mathrm{H}_{2} \mathrm{O}, \mathrm{C}_{2} \mathrm{H}_{2}$ in the case of monomers(Figure 7()). According to the fragmentation mechanism demonstrated, a total of 22 compounds are inferred (labeled as P13, 16, 17, 29, 42, 46, 48, $52,54,62,63,64,75,77,80,94,98,104,113,116,117,122)$.

3.1.4. Identification of Organic Acids. Organic acids are one kind of acidic compounds, which contain acidic functional groups, such as $\mathrm{COOH}, \mathrm{OH}, \mathrm{SO}_{3} \mathrm{H}, \mathrm{RSO}_{2} \mathrm{H}$, and $\mathrm{RCOSH}$. Because there is less $\mathrm{S}$ element in TCM, they are mainly 

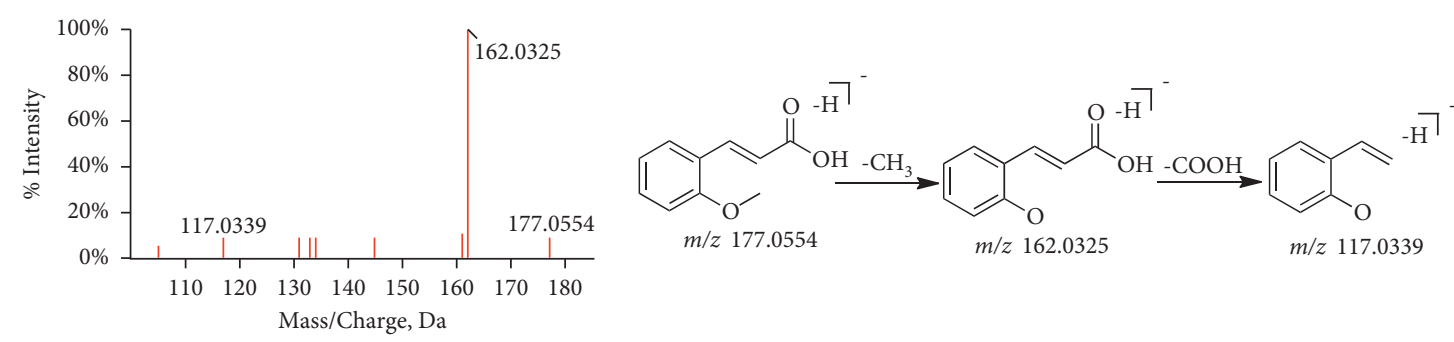

(a)

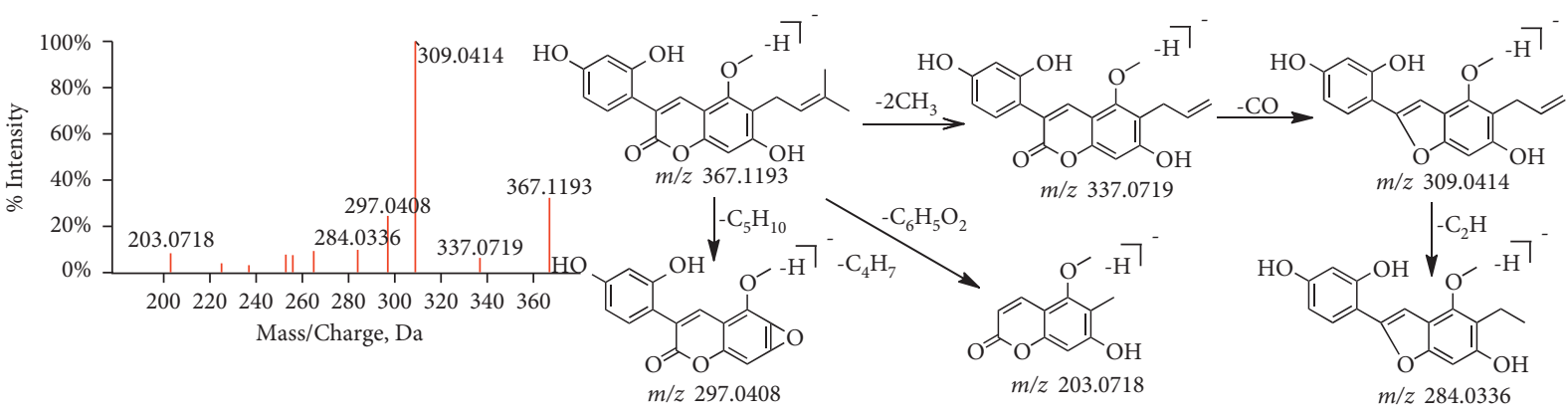

(b)
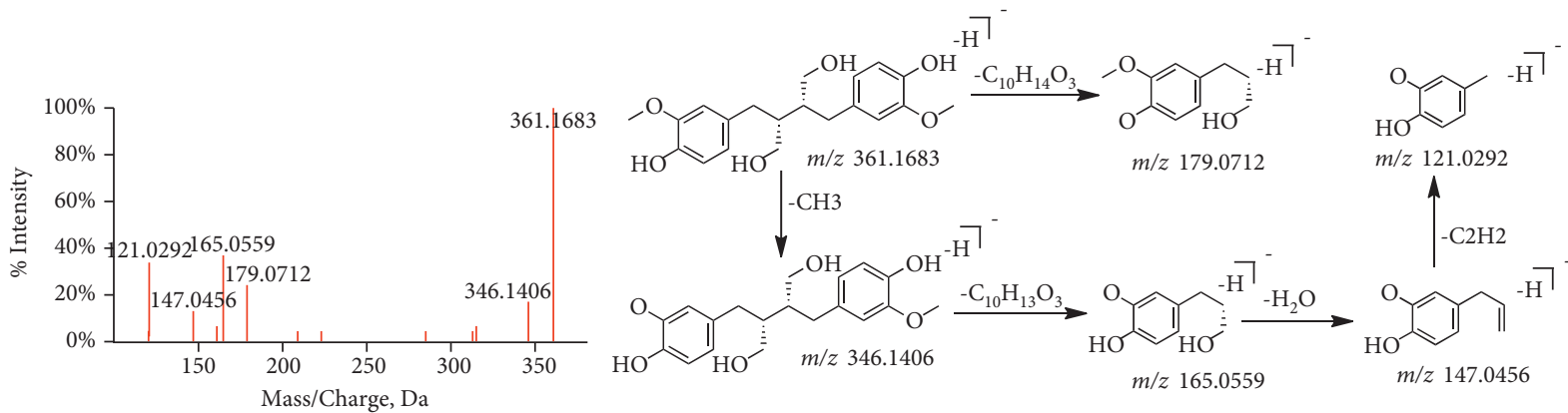

(c)

Figure 7: The fragmentation pathway of phenylpropanoids. The fragmentation pathway of (a) 2-methoxycinnamic acid, (b) glycycoumarin, and (c) secoisolariciresinol.

carboxylic acids and phenolic compounds. The response of these compounds in negative ion mode is better than that in positive, and it is easy to lose neutral molecules, such as $\mathrm{CH}_{3} \cdot \mathrm{H}_{2} \mathrm{O}, \mathrm{CH}_{3} \mathrm{O}, \mathrm{CO}_{2}$, and $\mathrm{COOH}$. When the compound contains ferulic acid or caffeic acid, the loss of feruloyl or caffeoyl $\mathrm{C}_{9} \mathrm{H}_{6} \mathrm{O}_{3}(146 \mathrm{Da})$ is also easy to occur $[24,25]$. The prominent ion of vanillic acid $\left(\mathrm{C}_{8} \mathrm{H}_{7} \mathrm{O}_{4}, \mathrm{~m} / z\right.$ 167.0342, $\left.[\mathrm{M}-\mathrm{H}]^{-}, \mathrm{P9}\right)$ produced a series of characteristic ion at $\mathrm{m} / z$ $136.0174,123.0449,108.0221$, which is caused by the loss of $\mathrm{CH}_{3} \mathrm{O}, \mathrm{CO}_{2}$, and $\mathrm{C}_{2} \mathrm{H}_{3} \mathrm{O}_{2}\left(\mathrm{CH}_{3} \mathrm{O}+\mathrm{CO}\right)$ on the basis of molecular ion peaks (Figure 8). According to the rules discussed above, a total of 13 compounds are inferred (labeled as $\mathrm{P} 2,3,9,10,11,12,14,15,19,25,26,36,79)$.

3.1.5. Identification of the Other Types. In addition, by comparing the existing literature at home and abroad, exact molecular weight, mass spectrometry characteristics, and other related data, the study also preliminarily identified or inferred 21 species, including 5 alkaloids (labeled as P5, 6, 18, 22, 27), 6 lactones(labeled as P7, 8,
23, 61, 96, 127), 4 anthraquinones (labeled as P33, 56, $57,78), 2$ polysaccharides(labeled as $\mathrm{P} 1,4)$, and 4 other components(labeled as P31, 32, 95, 147) [26-30]. Given the large differences in the amounts of the various components, they are hereby grouped into other categories.

3.2. Biomarkers Screening and Validation by Network Pharmacology and Components Absorbed into Blood. A total of 284 potential targets related to the 149 identified compounds were obtained from BATMAN-TCM (http://bionet.ncpsb. org.cn/batman-tcm/) databases(with the score cutoff $>20$ ). Through the keyword "Hyperlipidemias," a total of 1,691 disease targets were obtained from DrugBank (https://go. drugbank.com/), OMIM (https://omim.org/), GeneCards (https://www. genecards.org/), and DisGeNET (https:// www.disgenet.org/) databases. Through protein-protein interaction analysis, 71 targets with higher association were obtained as shown in Figure 9(a). The relevant parameters of the first 20 targets with a higher degree of integration are 


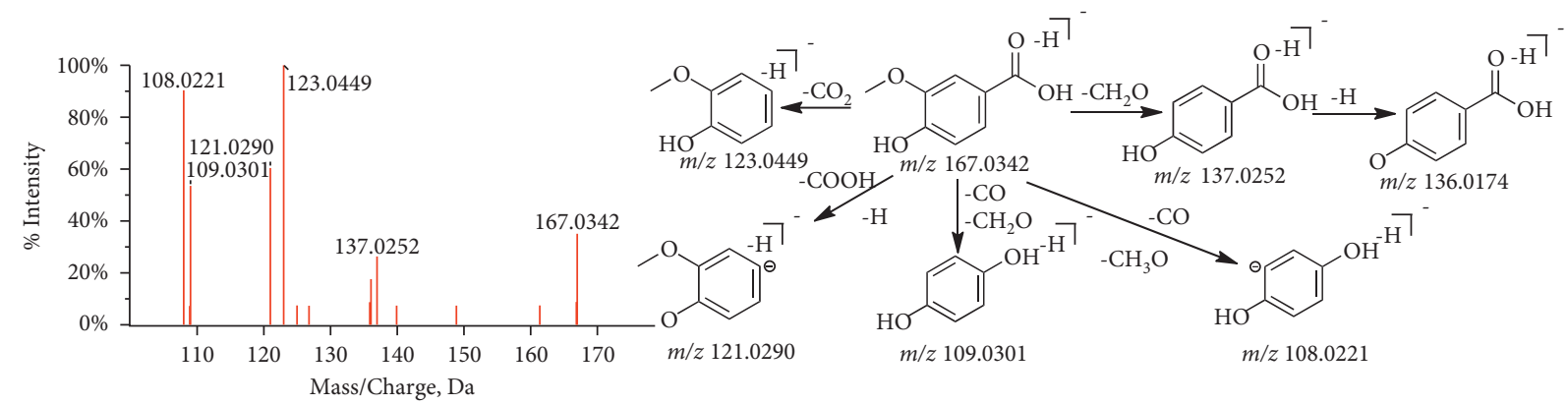

FIGURE 8: The fragmentation pathway of vanillic acid.

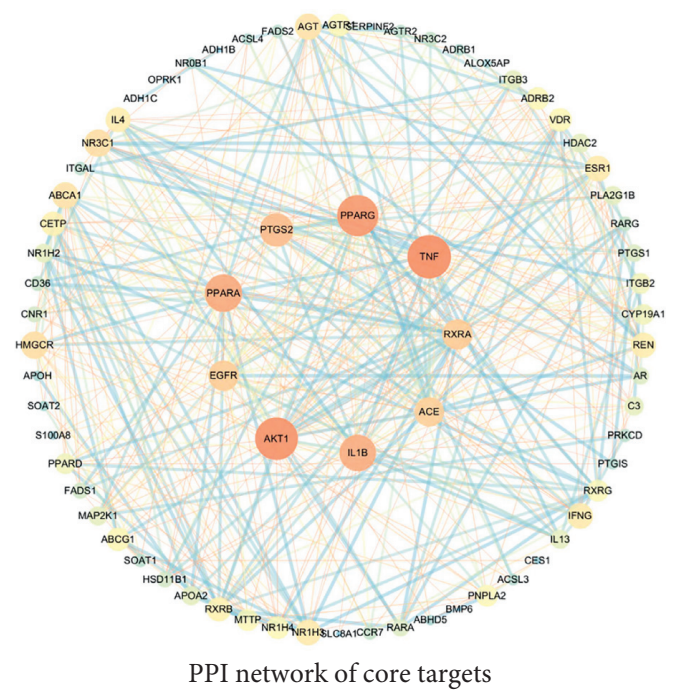

PPI network of core targets
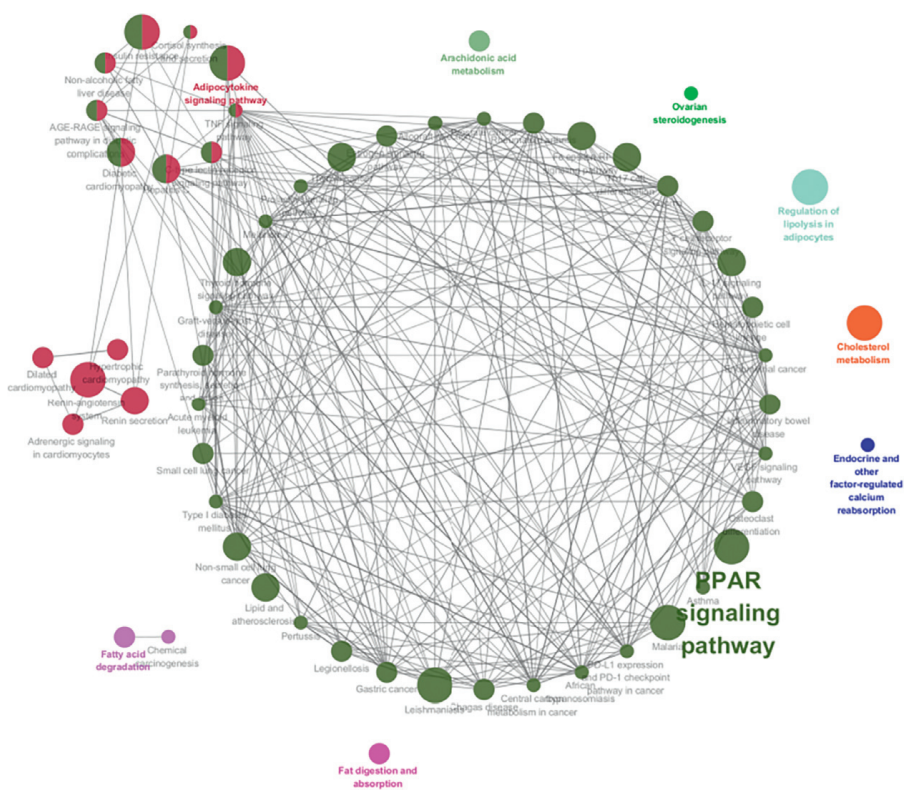

Results of KEGG pathway enrichment analysis

(a)

(b)

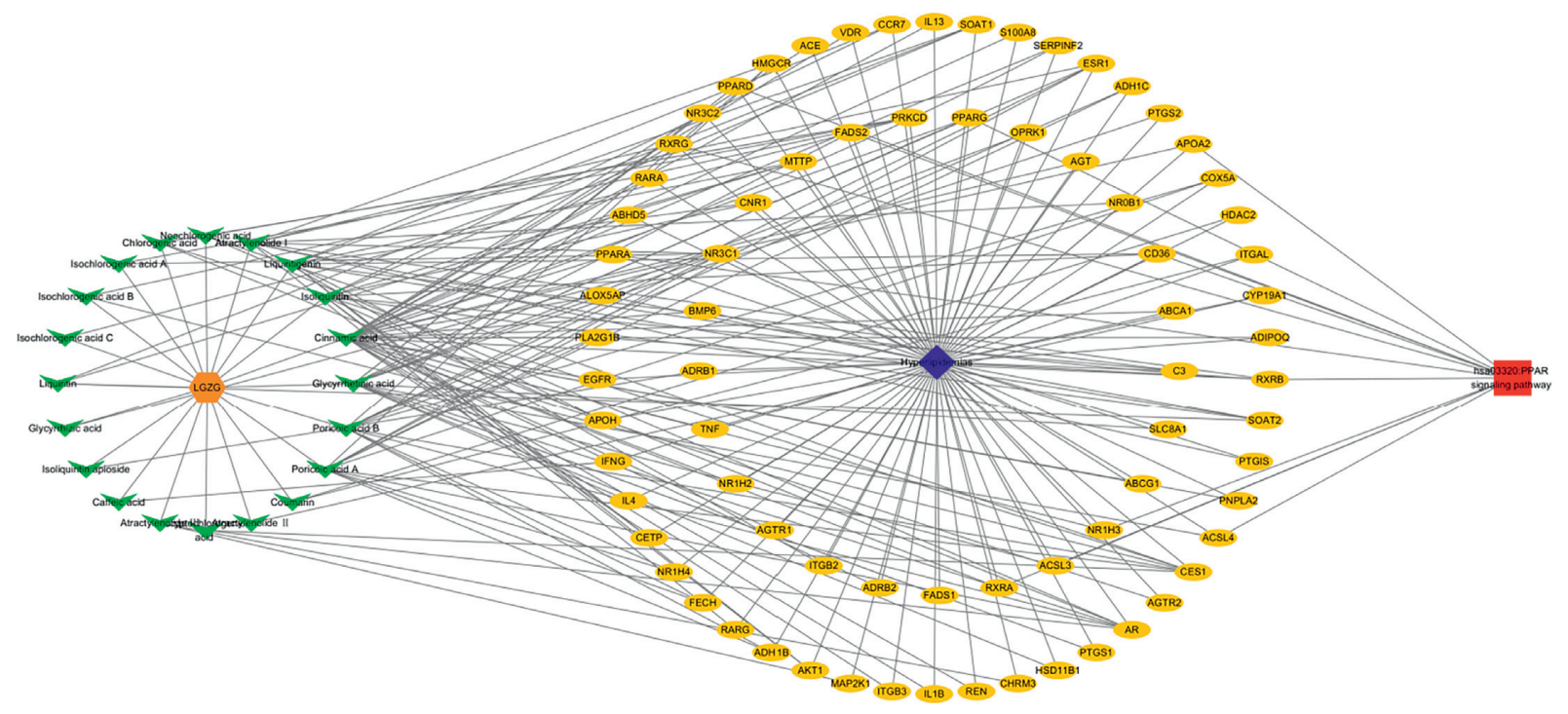

Component-Target-Function network diagram

(c)

FIGURE 9: Network pharmacology prediction analysis. (a) PPI network of core target. (b) Result of KEGG pathway enrichment analysis. (c) Component target function network diagram. 
TABLe 2: The first 20 key targets of LGZG decoction in treating hyperlipidemias.

\begin{tabular}{|c|c|c|c|c|}
\hline No. & Target name & Betweenness centrality & Closeness centrality & Degree \\
\hline 1 & TNF & 0.1205 & 0.7204 & 41 \\
\hline 2 & AKT1 & 0.1198 & 0.7053 & 40 \\
\hline 3 & PPARG & 0.1057 & 0.6907 & 38 \\
\hline 4 & PPARA & 0.0951 & 0.6569 & 33 \\
\hline 5 & IL1B & 0.0514 & 0.6505 & 32 \\
\hline 6 & PTGS2 & 0.0414 & 0.6091 & 28 \\
\hline 7 & RXRA & 0.0320 & 0.6036 & 24 \\
\hline 8 & EGFR & 0.0230 & 0.5877 & 24 \\
\hline 9 & $\mathrm{ACE}$ & 0.0371 & 0.5982 & 23 \\
\hline 10 & HMGCR & 0.0370 & 0.5776 & 20 \\
\hline 11 & NR3C1 & 0.0147 & 0.5492 & 20 \\
\hline 12 & ABCA1 & 0.0240 & 0.5678 & 19 \\
\hline 13 & AGT & 0.0320 & 0.5678 & 19 \\
\hline 14 & NR1H3 & 0.0090 & 0.5630 & 18 \\
\hline 15 & IFNG & 0.0144 & 0.5678 & 18 \\
\hline 16 & ESR1 & 0.0104 & 0.5194 & 18 \\
\hline 17 & REN & 0.0139 & 0.5537 & 17 \\
\hline 18 & IL4 & 0.0105 & 0.5583 & 17 \\
\hline 19 & CETP & 0.0142 & 0.5194 & 16 \\
\hline 20 & RXRB & 0.0072 & 0.5276 & 16 \\
\hline
\end{tabular}

shown in Table 2. These reserved proteins were further imported into the ClueGo plugin of Cytoscape 3.7.2 software for KEGG enrichment analysis, and relatively important disease pathways were found as shown in Figure 9(b). Finally, the "component-target-function" network was visualized in Figure 9(c). There are a total of 20 compounds (including poricoic acid A, poricoic acid B, glycyrrhizic acid, glycyrrhetinic acid, liquiritin, isoliquiritin, liquiritigenin, isoliquiritin apioside, cinnamic acid, caffeic acid, neochlorogenic acid, chlorogenic acid, cryptochlorogenic acid, isochlorogenic acid $\mathrm{A}$, isochlorogenic acid $\mathrm{B}$, isochlorogenic acid C, atractylenolide I, atractylenolide II, atractylenolide III, and coumarin), 71 proteins, and 20 pathways involved. Of the 20 pathways, the score of PPAR pathway was the highest, which plays an important role in lipid metabolism. On this basis, we verify it by the method of molecular docking. The results show that the free energies of docking binding of 20 compounds are all greater than $-6.471 \mathrm{kcal} /$ mol. The typical components of the four TCM we selected are displayed in Figure 10. Besides, all the 20 compounds were detected in the rat serum by using UPLC-QQQ-MS/ MS, which further verified that these compounds were proper biomarkers.

3.3. Quantification of the Major Constituents in LGZG by $U P L C-Q Q Q-M S / M S$. On the basis of the qualitative study and network pharmacology, the characteristic parameters of 20 main chemical components were investigated by the MRM analysis method, including parent ion, daughter ion, declustering potential, and collision energy. In order to satisfy the simultaneous quantification of multiple components, taking into account the response of different chemical components in MRM mode and the content differences in LGZG, the DP and CE of some chemical components were dynamically adjusted, as detailed in Table 3 [31-37]. Multiple reaction monitoring chromatograms of the control and sample solutions are shown in Figure 11.

\subsubsection{Method Validation}

(1) Calibration Curve, LOD, and LOQ. According to the concentration $(\mathrm{X})$ and peak area $(\mathrm{Y})$ of each reference substance, the calibration curves were drawn and linear regressions were carried out, which showed good linearities $\left(\mathrm{R}^{2}>0.9937\right)$. In addition, the LOD and LOQ values were determined, and the obtained results are presented in Table 4.

(2) Precision, Repeatability, Stability, and Recovery. The precision, repeatability, stability, and recovery of the quantitative method were investigated. The RSD of the precision of the reference substance and the sample did not exceed $4.29 \%$ and $5.02 \%$, respectively. The repetitive result is less than $6.47 \%$. The stability test results show that the LGZG sample solution is stable within 48 hours, and the RSD is less than $6.01 \%$. The average recovery is between $96.22 \%$ and $104.19 \%$. The results show that the established quantitative method is accurate, reliable, and reproducible and can be used to evaluate the quality of LGZG (Table 5).

3.3.2. Sample Analysis. The established quantitative method was used for the determination of 20 compounds in 18 batches of LGZG from different producing areas. The content ranges were poricoic acid A $0.46 \sim 1.09 \mu \mathrm{g} / \mathrm{g}$, poricoic acid B $0.53 \sim 1.43 \mu \mathrm{g} / \mathrm{g}$, glycyrrhizic acid $47.06 \sim 60.36 \mathrm{mg} / \mathrm{g}$, glycyrrhetinic acid $1.07 \sim 3.32 \mu \mathrm{g} / \mathrm{g}$, liquiritin $2.03 \sim 6.13 \mathrm{mg} / \mathrm{g}$, isoliquiritin $204.17 \sim 428.91 \mu \mathrm{g} / \mathrm{g}$, liquiritigenin $0.72 \sim 1.30 \mathrm{mg} / \mathrm{g}$, isoliquiritin apioside $0.82 \sim 3.25 \mathrm{mg} / \mathrm{g}$, cinnamic acid $0.58 \sim 1.17 \mu \mathrm{g} / \mathrm{g}$, caffeic acid $6.07 \sim 24.93 \mu \mathrm{g} / \mathrm{g}$, neochlorogenic acid $24.33 \sim 252.05 \mu \mathrm{g} / \mathrm{g}$, chlorogenic acid 109.42-1386.51 $\mu \mathrm{g} / \mathrm{g}$, 


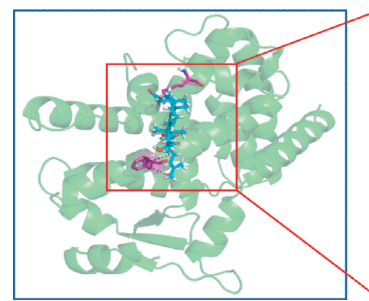

Poricoic acid B with PPAR $\alpha$ (blinding energy $=-6.670 \mathrm{kcal} / \mathrm{mol}$ )

(a)

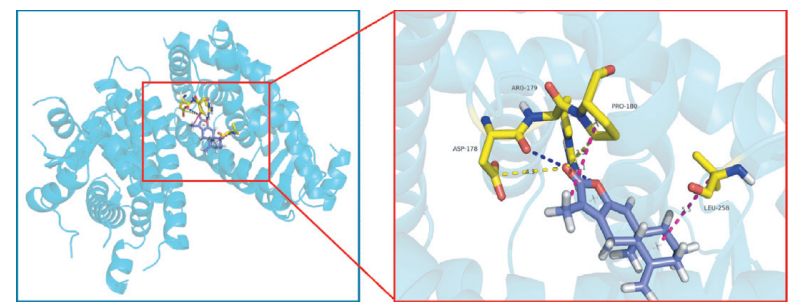

Atractylenolide I with PPAR $\gamma$ (blinding energy $=-7.949 \mathrm{kcal} / \mathrm{mol}$ )

(c)

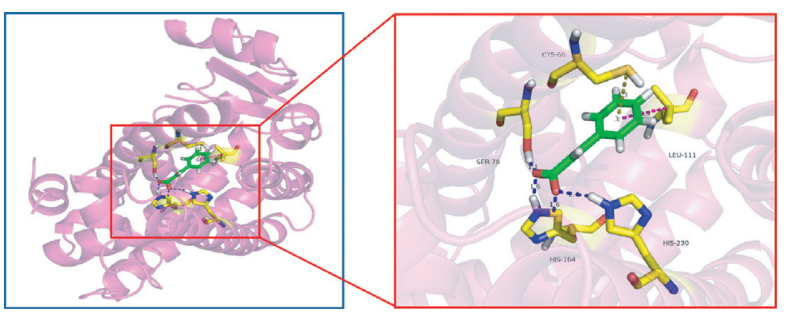

Cinnamic acid with PPAR $\alpha$ (blinding energy $=-7.186 \mathrm{kcal} / \mathrm{mol}$ )

(b)

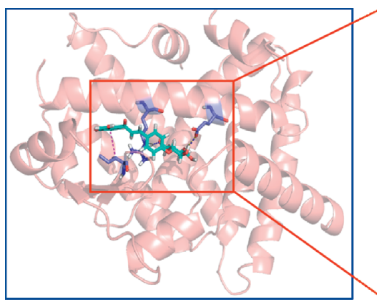

Isoliquiritin with $\mathrm{PPAR} \alpha$

(blinding energy $=-9.397 \mathrm{kcal} / \mathrm{mol}$ )

(d)

FIGURE 10: Result of molecular docking between typical chemical composition. (a) Poricoic acid B with PPAR $($ binding energy $=-6.670 \mathrm{kcal} / \mathrm{mol}$ ).

(b) Cinnamic acid with PPAR $\alpha$ (binding energy $=-7.186 \mathrm{kcal} / \mathrm{mol}$ ). (c) Atractylenolide I with PPAR $\gamma$ (binding energy $=-7.949 \mathrm{kcal} / \mathrm{mol}$ ).

(d) Isoliquiritin with PPAR $\alpha$ (binding energy $=-9.397 \mathrm{kcal} / \mathrm{mol}$ ).

TABLE 3: Mass spectrometry data of 20 chemical constituents.

\begin{tabular}{|c|c|c|c|c|c|c|}
\hline Chemical components & $\begin{array}{c}t_{R} \\
(\mathrm{~min})\end{array}$ & $\begin{array}{c}\text { Detection } \\
\text { mode }\end{array}$ & $\begin{array}{c}\text { Parent ion } \\
(\mathrm{m} / z)\end{array}$ & $\begin{array}{l}\text { Daughter ion } \\
(\mathrm{m} / z)\end{array}$ & $\begin{array}{c}\text { Declustering potential } \\
(\mathrm{V})\end{array}$ & $\begin{array}{c}\text { Collision energy } \\
(\mathrm{V})\end{array}$ \\
\hline Poricoic acid A & 31.36 & $\mathrm{M}-\mathrm{H}$ & 497.2 & 423.3 & -44.00 & -214.00 \\
\hline Poricoic acid B & 30.64 & $\mathrm{M}-\mathrm{H}$ & 483.4 & 409.4 & -44.40 & -203.00 \\
\hline Glycyrrhizic acid & 25.53 & $\mathrm{M}-\mathrm{H}$ & 821.4 & 350.8 & -78.00 & -276.00 \\
\hline Glycyrrhetinic acid & 31.80 & $\mathrm{M}-\mathrm{H}$ & 469.2 & 425.4 & -49.90 & -247.00 \\
\hline $\begin{array}{l}\text { Liquiritin } \\
\text { And isoliquiritin }\end{array}$ & $\begin{array}{l}17.81 \\
22.31\end{array}$ & $\mathrm{M}-\mathrm{H}$ & 417.2 & 255.0 & -47.00 & -215.00 \\
\hline Liquiritigenin & 17.81 & $\mathrm{M}-\mathrm{H}$ & 255.0 & 134.7 & -43.00 & -178.00 \\
\hline Isoliquiritin apioside & 22.04 & $\mathrm{M}-\mathrm{H}$ & 549.2 & 254.8 & -53.00 & -284.00 \\
\hline Cinnamic acid & 23.23 & $\mathrm{M}-\mathrm{H}$ & 146.6 & 102.8 & -14.08 & -148.86 \\
\hline Caffeic acid & 9.31 & $\mathrm{M}-\mathrm{H}$ & 178.6 & 134.6 & -23.88 & -104.01 \\
\hline $\begin{array}{l}\text { Neochlorogenic acid and } \\
\text { chlorogenic acid }\end{array}$ & $\begin{array}{l}5.02 \\
8.35\end{array}$ & $\mathrm{M}-\mathrm{H}$ & 353.0 & 190.6 & -22.91 & -152.04 \\
\hline Cryptochlorogenic acid & $\begin{array}{c}8.91 \\
20.96\end{array}$ & $\mathrm{M}-\mathrm{H}$ & 353.1 & 172.6 & -22.43 & -114.04 \\
\hline Isochlorogenic acid $\mathrm{A}, \mathrm{B}$, and $\mathrm{C}$ & $\begin{array}{l}20.59 \\
21.54\end{array}$ & M-H & 515.1 & 353.1 & -37.00 & -180.00 \\
\hline Atractylenolide III & 27.76 & $\mathrm{M}-\mathrm{H}$ & 247.0 & 202.7 & -22.95 & -147.87 \\
\hline Atractylenolide I & 31.56 & $\mathrm{M}+\mathrm{H}$ & 231.1 & 129.0 & 37.83 & 136.00 \\
\hline Atractylenolide II & 29.98 & $\mathrm{M}+\mathrm{H}$ & 233.3 & 215.2 & 20.00 & 133.00 \\
\hline Coumarin & 19.70 & $\mathrm{M}+\mathrm{H}$ & 147.2 & 91.0 & 28.03 & 109.00 \\
\hline
\end{tabular}

cryptochlorogenic acid 25.65 217.65 $\mu \mathrm{g} / \mathrm{g}$, isochlorogenic acid A $20.98 \sim 68.03 \mu \mathrm{g} / \mathrm{g}$, isochlorogenic acid B $75.52 \sim 193.39 \mu \mathrm{g} / \mathrm{g}$, isochlorogenic acid C 34.37 92.05 $\mu \mathrm{g} / \mathrm{g}$, atractylenolide I $6.77 \sim 25.56 \mu \mathrm{g} / \mathrm{g}$, atractylenolide II $16.95 \sim 124.62 \mu \mathrm{g} / \mathrm{g}$, atractylenolide III $58.34 \sim 339.74 \mu \mathrm{g} / \mathrm{g}$, and coumarin
281.17 410.14 $\mu \mathrm{g} / \mathrm{g}$ (Figure 12, Table 6). The established determination method has been confirmed to be simple in operation, which also has the advantages of good precision, stability, repeatability, and recovery. It can provide a reference for the quality evaluation and quality control of the LGZG. 


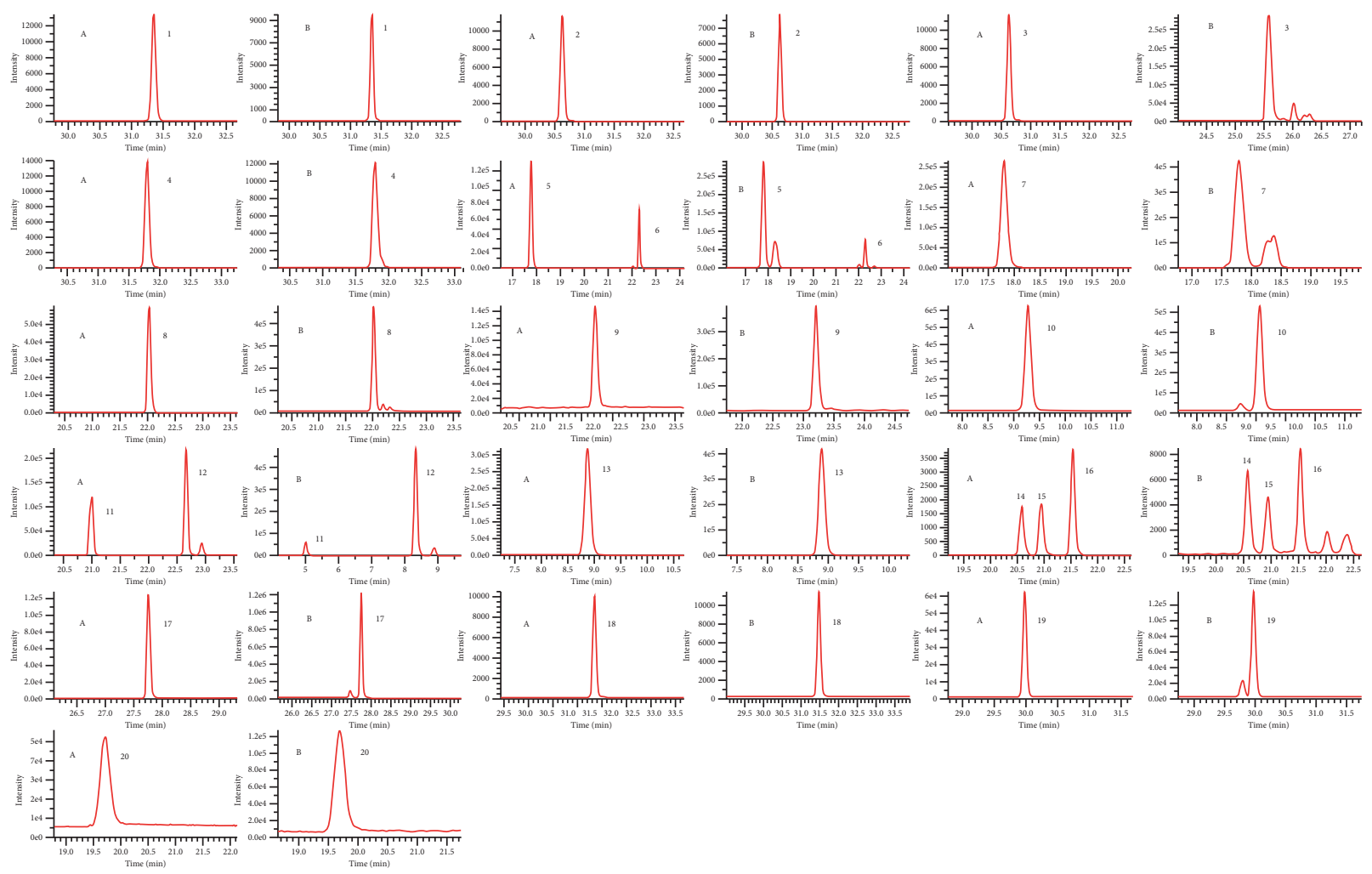

FIGURE 11: MRM chromatograms of reference substances (A) and samples (B). (1) Poricoic acid (A); (2) poricoic acid (B); (3) glycyrrhizic acid; (4) glycyrrhetinic acid; (5) liquiritin; (6) isoliquiritin; (7) liquiritigenin; (8) isoliquiritin apioside; (9) cinnamic acid; (10) caffeic acid; (11) neochlorogenic acid; (12) chlorogenic acid; (13) cryptochlorogenic acid; (14) isochlorogenic acid; (15) isochlorogenic acid (A); (16) isochlorogenic acid (C); (17) atractylenolide III; (18) atractylenolide (I); (19) atractylenolide II; (20) coumarin.

TABLE 4: Regression equations, correlation coefficients, linear ranges, LODs, and LOQs of 20 chemical constituents.

\begin{tabular}{|c|c|c|c|c|c|}
\hline $\begin{array}{l}\text {-Col Count:6-->Chemical components } \\
\end{array}$ & Calibration curve & $R^{2}$ & Linearity range $(\mu \mathrm{g} / \mathrm{mL})$ & LOD $(\mathrm{ng} / \mathrm{mL})$ & LOQ $(\mathrm{ng} / \mathrm{mL})$ \\
\hline Poricoic acid A & $Y=72391 X-5034.7$ & 0.9994 & $0.002 \sim 0.21$ & 0.199 & 0.665 \\
\hline Poricoic acid B & $Y=68768 X-7167.6$ & 0.9989 & $0.002 \sim 0.22$ & 0.177 & 0.591 \\
\hline Glycyrrhizic acid & $Y=229221 X-89186$ & 0.9970 & $2.829 \sim 282.9$ & 499.2 & 1664 \\
\hline Glycyrrhetinic acid & $Y=76305 X+1723.8$ & 0.9995 & $0.003 \sim 0.32$ & 0.461 & 1.538 \\
\hline Liquiritin & $Y=1 \mathrm{E}+06 \mathrm{X}-97606$ & 0.9952 & $3.264 \sim 326.4$ & 90.08 & 300.3 \\
\hline Isoliquiritin & $Y=472548 X-40833$ & 0.9988 & $0.042 \sim 42.12$ & 12.74 & 42.46 \\
\hline Liquiritigenin & $Y=1 \mathrm{E}+06 \mathrm{X}+1 \mathrm{E}+06$ & 0.9959 & $2.465 \sim 61.63$ & 28.94 & 96.48 \\
\hline Isoliquiritin apioside & $Y=601173 X-225511$ & 0.9950 & $0.536 \sim 53.62$ & 26.81 & 89.37 \\
\hline Cinnamic acid & $Y=751463 X+182882$ & 0.9939 & $0.768 \sim 76.80$ & 157.8 & 526.0 \\
\hline Caffeic acid & $Y=5 E+06 X+1 E+06$ & 0.9945 & $0.017 \sim 1.697$ & 1.858 & 6.192 \\
\hline Neochlorogenic acid & $Y=3 \mathrm{E}+06 \mathrm{X}-953535$ & 0.9963 & $0.166 \sim 16.55$ & 5.623 & 18.72 \\
\hline Chlorogenic acid & $Y=4 \mathrm{E}+06 \mathrm{X}-961985$ & 0.9978 & $0.162 \sim 16.15$ & 2.525 & 8.416 \\
\hline Cryptochlorogenic acid & $Y=3 \mathrm{E}+06 \mathrm{X}+88218$ & 0.9960 & $0.055 \sim 5.528$ & 1.229 & 4.096 \\
\hline Isochlorogenic acid $\mathrm{A}$ & $Y=31732 X-10255$ & 0.9971 & $0.039 \sim 3.894$ & 4.314 & 14.38 \\
\hline Isochlorogenic acid B & $Y=33.14 \mathrm{X}+17963$ & 0.9997 & $0.024 \sim 2.430$ & 8.404 & 28.01 \\
\hline Isochlorogenic acid C & $Y=48826 X-11262$ & 0.9937 & $0.034 \sim 3.353$ & 7.288 & 24.29 \\
\hline Atractylenolide III & $Y=628413 X-27614$ & 0.9996 & $0.060 \sim 6.000$ & 3.379 & 11.26 \\
\hline Atractylenolide I & $Y=78592 X-1874.8$ & 0.9995 & $0.033 \sim 3.300$ & 18.80 & 62.67 \\
\hline Atractylenolide II & $Y=345026 X+43919$ & 0.9986 & $0.094 \sim 9.400$ & 13.95 & 46.51 \\
\hline Coumarin & $Y=731434 X-14528$ & 0.9998 & $0.274 \sim 27.36$ & 241.41 & 804.7 \\
\hline
\end{tabular}


TABLE 5: The results of precision, reproducibility, stability, and recovery of 20 chemical constituents.

\begin{tabular}{|c|c|c|c|c|c|c|}
\hline \multirow{2}{*}{ Chemical components } & \multicolumn{2}{|c|}{ Precision (RSD/\%) } & \multirow{2}{*}{ Reproducibility (RSD/\%) } & \multirow{2}{*}{ Stability (RSD/\%) } & \multicolumn{2}{|c|}{ Recovery } \\
\hline & Reference & Sample & & & Average (\%) & RSD (\%) \\
\hline Poricoic acid $\mathrm{A}$ & 1.77 & 4.22 & 0.81 & 4.42 & 99.25 & 2.42 \\
\hline Poricoic acid B & 2.70 & 2.96 & 2.83 & 5.41 & 102.24 & 1.06 \\
\hline Glycyrrhizic acid & 1.25 & 1.36 & 3.28 & 4.79 & 103.39 & 3.23 \\
\hline Glycyrrhetinic acid & 2.85 & 2.95 & 3.70 & 4.25 & 98.24 & 1.46 \\
\hline Liquiritin & 2.44 & 2.27 & 5.54 & 5.16 & 100.30 & 2.60 \\
\hline Isoliquiritin & 3.55 & 2.44 & 2.16 & 4.29 & 99.75 & 1.84 \\
\hline Liquiritigenin & 1.80 & 0.57 & 2.45 & 1.52 & 96.22 & 3.11 \\
\hline Isoliquiritin apioside & 3.67 & 1.83 & 5.51 & 1.98 & 100.95 & 1.69 \\
\hline Cinnamic acid & 2.94 & 3.38 & 2.79 & 5.10 & 99.66 & 2.21 \\
\hline Caffeic acid & 2.17 & 1.07 & 3.42 & 2.60 & 102.35 & 1.50 \\
\hline Neochlorogenic acid & 2.83 & 1.69 & 1.53 & 5.24 & 98.99 & 1.83 \\
\hline Chlorogenic acid & 3.40 & 3.46 & 2.40 & 5.78 & 96.98 & 2.41 \\
\hline Cryptochlorogenic acid & 2.27 & 1.54 & 3.94 & 4.98 & 99.61 & 1.99 \\
\hline Isochlorogenic acid A & 1.60 & 4.42 & 2.99 & 2.72 & 104.19 & 1.88 \\
\hline Isochlorogenic acid B & 4.29 & 3.75 & 2.44 & 3.73 & 96.84 & 2.99 \\
\hline Isochlorogenic acid C & 4.07 & 1.79 & 4.70 & 5.55 & 102.26 & 1.34 \\
\hline Atractylenolide III & 2.78 & 3.54 & 3.34 & 3.86 & 101.39 & 1.45 \\
\hline Atractylenolide I & 3.23 & 5.02 & 6.15 & 6.01 & 99.90 & 2.81 \\
\hline Atractylenolide II & 1.95 & 2.08 & 5.79 & 6.00 & 97.82 & 1.22 \\
\hline Coumarin & 3.73 & 2.22 & 6.47 & 5.98 & 98.13 & 1.16 \\
\hline
\end{tabular}

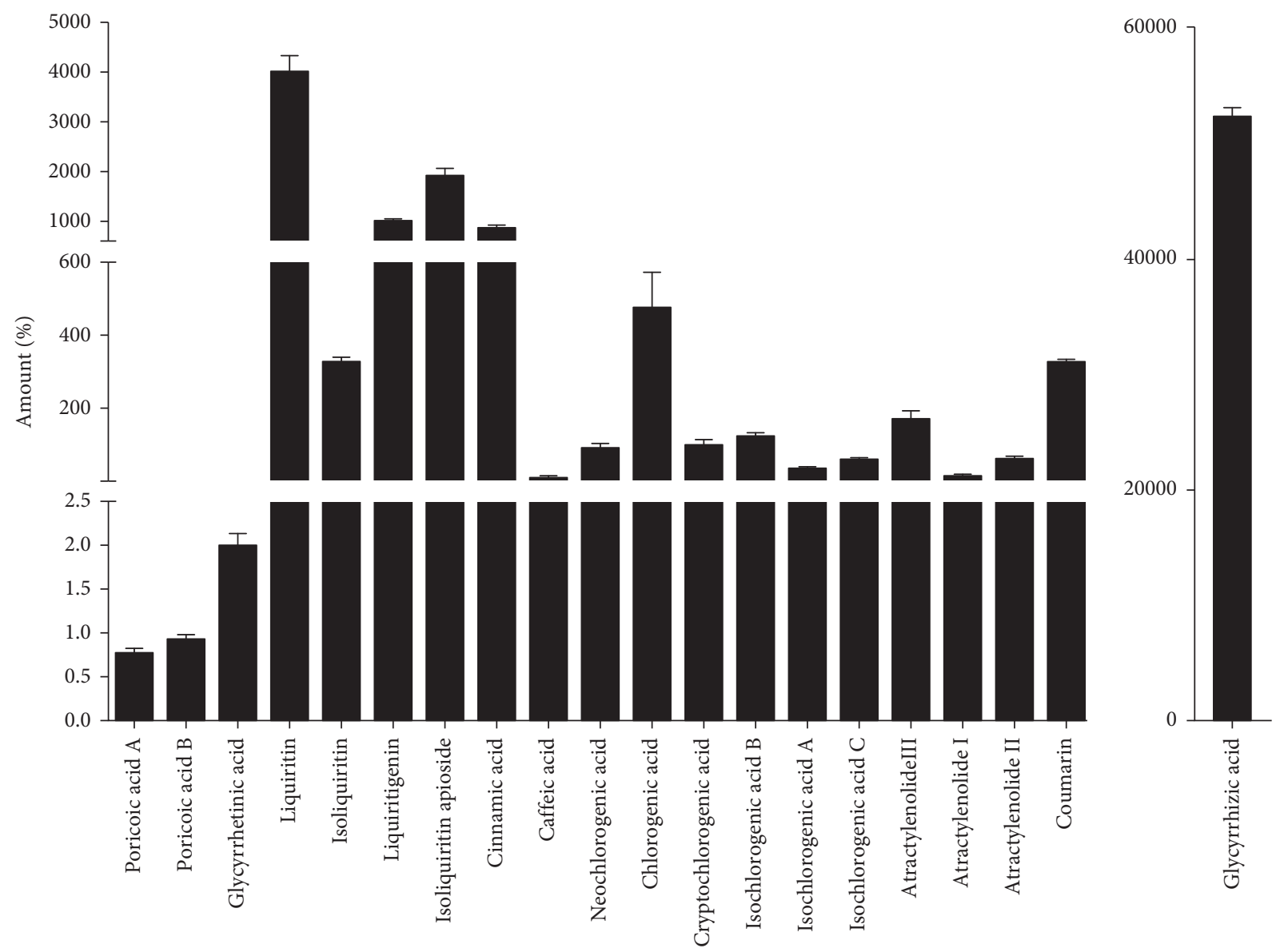

(a)

Figure 12: Continued. 


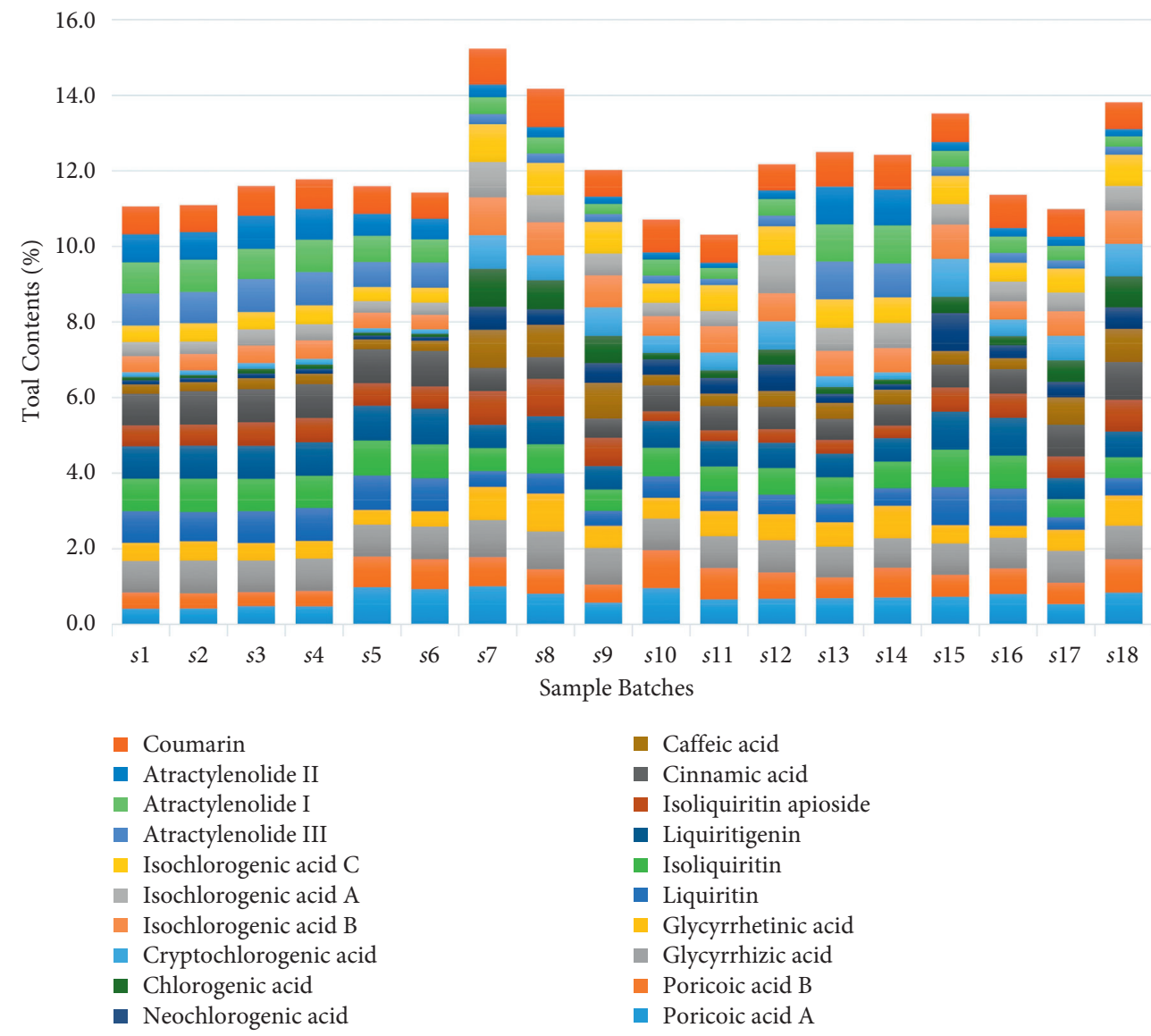

(b)

FIgUre 12: The quantitative study analysis results of LGZC. (a) Quantification results of 20 chemical components. (b) Total contents differences of 20 chemical components among the 18 batches. 


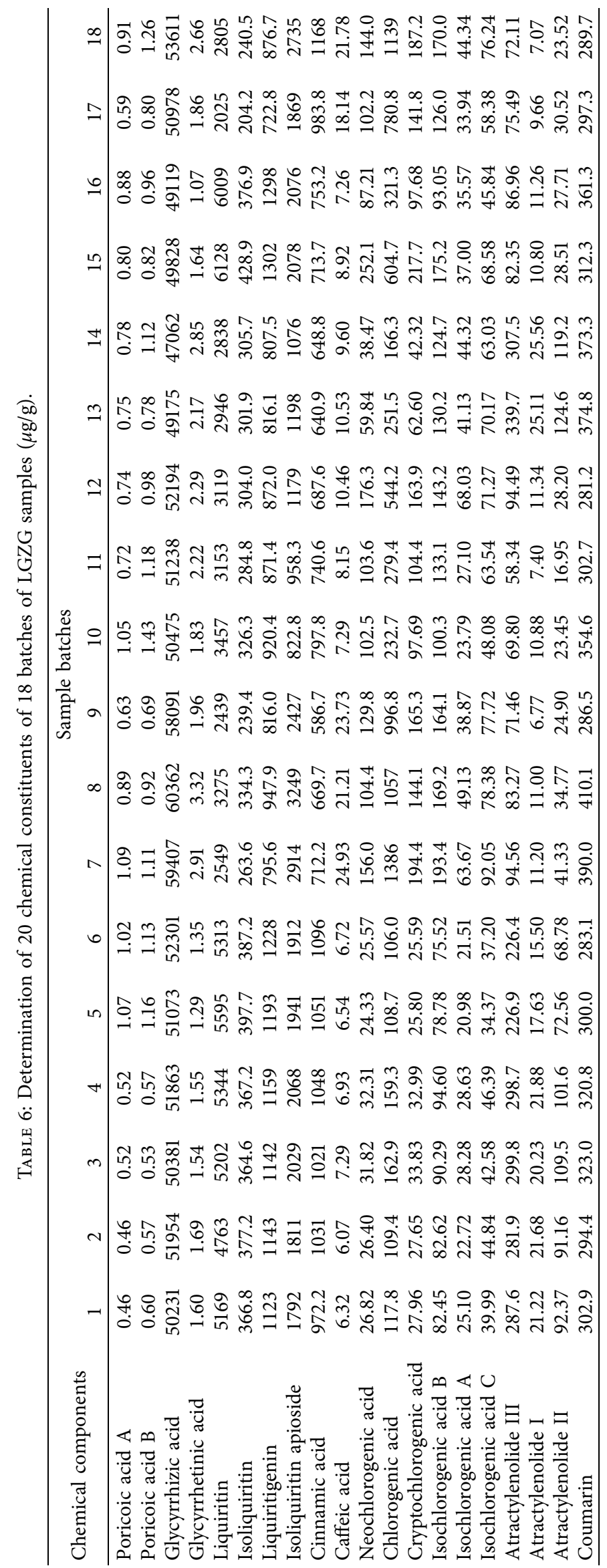




\section{Conclusion}

Based on UPLC-MS/MS and network pharmacology, this article established a research method of chemical composition research-network pharmacology predictive analysisquality evaluation system. First of all, the 149 components of LGZG were preliminarily identified or inferred by UPLC-Q/ TOF-MS, including flavonoids, triterpenoids, phenylpropanoids, organic acids, quinones, and other types of components. Then, a total of 20 biomarkers were screened by network pharmacological screening based on the presumed chemical composition. Finally, the quantitative determination method of biomarkers in LGZG was established by UPLC-QQQ-MS/MS, which covered all 4 herbal medicines in LGZG. Combined with statistical analysis, the quality evaluation system of LGZG was established. Following the results of this study, its pharmacological effects point to lipid metabolism-related targets and pathways. Therefore, the experimental group intends to carry out further studies on LGZG metabolomics and lipidomics to provide further data support for its pharmacodynamics and pharmacological mechanism.

\section{Data Availability}

The figure and table data used to support the findings of this study are included within the article.

\section{Conflicts of Interest}

The authors declare that they have no conflicts of interest.

\section{Authors' Contributions}

Baolin Li and Shuaishuai Fan contributed equally to this work.

\section{Acknowledgments}

This work was supported by the Central Government Guides Local Science and Technology Development Fund Projects (No. 206Z2501G); Hebei Provincial Key Research and Development Projects (No. 20372502D); Natural Science Foundation of Hebei Province (No. H2019423050); and Scientific Research Project of Hebei Administration of Traditional Chinese Medicine (No. 2021100).

\section{Supplementary Materials}

Table S1: characterization of chemical components derived from LGZG by UPLC-Q/TOF-MS/MS. (Supplementary Materials)

\section{References}

[1] X. Li, G. Xu, and S. Wei, "Lingguizhugan decoction attenuates doxorubicin-induced heart failure in rats by improving TT-SR microstructural remodeling," BMC Complementary and Alternative Medicine, vol. 19, no. 1, p. 360, 2019.

[2] R. Wu, D. Zhao, and R. An, "Linggui zhugan formula improves glucose and lipid levels and alters gut microbiota in high-fat diet-induced diabetic mice," Frontiers in Physiology, vol. 10, p. 918, 2019.

[3] F. Xi, F. Sang, C. Zhou, and Y. Ling, "Protective effects of Lingguizhugan decoction on amyloid-beta peptide (25-35)-induced cell injury: anti-inflammatory effects," Neural Regeneration Research, vol. 7, no. 36, pp. 2867-2873, 2012.

[4] X. Wang, Y. Gao, and Y. Tian, "Integrative serum metabolomics and network analysis on mechanisms exploration of Ling-Gui-Zhu-Gan Decoction on doxorubicin-induced heart failure mice," Journal of Ethnopharmacology, vol. 250, Article ID 112397, 2020.

[5] L. Wang, H. Shi, J. L. Huang, S. Xu, and P. P. Liu, "Linggui zhugan decoction () inhibits ventricular remodeling after acute myocardial infarction in mice by suppressing TGF- $\beta(1) /$ smad signaling pathway," Chinese Journal of Integrative Medicine, vol. 26, no. 5, pp. 345-352, 2020.

[6] J. Xu, R. Wang, and S. You, "Traditional Chinese medicine Lingguizhugan decoction treating non-alcoholic fatty liver disease with spleen-yang deficiency pattern: study protocol for a multicenter randomized controlled trial," Trials, vol. 21, no. 1, p. 512, 2020.

[7] M. T. Liu, Y. J. Huang, T. Y. Zhang, L. B. Tan, X. F. Lu, and J. Qin, "Lingguizhugan decoction attenuates diet-induced obesity and hepatosteatosis via gut microbiota," World Journal of Gastroenterology, vol. 25, no. 27, pp. 3590-3606, 2019.

[8] L. Yao, J. Wei, and S. Shi, "Modified lingguizhugan decoction incorporated with dietary restriction and exercise ameliorates hyperglycemia, hyperlipidemia and hypertension in a rat model of the metabolic syndrome," BMC Complementary and Alternative Medicine, vol. 17, no. 1, p. 132, 2017.

[9] X. Hou, C. Wang, and Y. Li, "Clinical efficacy of Linggui Zhugan decoction in the treatment of decreased gastric motility caused by chronic heart failure," Minerva Medica, 2021.

[10] H. Ma, L. Guo, and Y. Chen, "Linggui Zhugan Decoction for peripheral vertigo: a protocol for systematic review and metaanalysis," Medicine (Baltimore), vol. 100, no. 16, p. e25563, 2021.

[11] S. Y. Xiang, J. Zhao, and Y. Lu, "Network pharmacology-based identification for therapeutic mechanism of Ling-Gui-ZhuGan decoction in the metabolic syndrome induced by antipsychotic drugs," Computers in Biology and Medicine, vol. 110, pp. 1-7, 2019.

[12] X. Y. Li, "Chemical profile Analysis of ling-gui-zhu-Gan decoction by LC-QTOF MS and simultaneous determination of nine major components using QAMS method," Chromatographia, vol. 83, no. 11, pp. 1371-1389, 2019.

[13] B. Ji, Y. Zhao, P. Yu, B. Yang, C. Zhou, and Z. Yu, "LC-ESI-MS/MS method for simultaneous determination of eleven bioactive compounds in rat plasma after oral administration of Ling-GuiZhu-Gan Decoction and its application to a pharmacokinetics study," Talanta, vol. 190, pp. 450-459, 2018.

[14] N. Li, L. Xie, and N. Yang, "Rapid classification and identification of chemical constituents in Epimedium koreanum Nakai by UPLC-Q-TOF-MS combined with data post-processing techniques," Phytochemical Analysis, vol. 32, no. 4, pp. 575-591, 2021.

[15] Y. Tong, Y. Jiang, and X. Chen, "Extraction, enrichment, and quantification of main antioxidant aglycones of flavonoids and tannins from Melastoma dodecandrum lour.: guided by UPLC-ESI-MS/MS," Journal of Chemistry, vol. 2019, Article ID 2793058, 12 pages, 2019.

[16] Y. Dong, G. Jia, and J. Hu, "Determination of alkaloids and flavonoids in Sophora flavescens by UHPLC-Q-TOF/MS," 
Journal of Analytical Methods in Chemistry, vol. 2021, Article ID 9915027, 13 pages, 2021.

[17] X. L. Qiu and Q. F. Zhang, "Identification and quantification of main flavonoids in the leaves of Bambusa multiplex cv. Fernleaf," Natural Product Research, vol. 34, no. 14, pp. 2076-2079, 2020.

[18] H. Xin, L. Fang, and J. Xie, "Identification and quantification of triterpenoids in lingzhi or reishi medicinal mushroom, ganoderma lucidum (agaricomycetes), with HPLC-MS/MS methods," International Journal of Medicinal Mushrooms, vol. 20, no. 10, pp. 919-934, 2018.

[19] Q. Huang, F. Zhang, S. Liu, Y. Jiang, and D. Ouyang, "Systematic investigation of the pharmacological mechanism for renal protection by the leaves of Eucommia ulmoides Oliver using UPLC-Q-TOF/MS combined with network pharmacology analysis," Biomedicine \& Pharmacotherapy, vol. 140, Article ID 111735, 2021.

[20] F. Wang, S. Huang, and Q. Chen, "Chemical characterisation and quantification of the major constituents in the Chinese herbal formula Jian-Pi-Yi-Shen pill by UPLC-Q-TOF-MS/MS and HPLC-QQQ-MS/MS," Phytochemical Analysis, vol. 31, no. 6, pp. 915-929, 2020.

[21] J. Yao, H. He, and J. Xue, "Mori ramulus (Chin.Ph.)-the dried twigs of morus alba L./Part 1: discovery of two novel coumarin glycosides from the anti-hyperuricemic ethanol extract," Molecules, vol. 243 pages, 2019.

[22] B. Zhuang, Z. M. Bi, Z. Y. Wang, L. Duan, C. J. Lai, and E. H. Liu, "Chemical profiling and quantitation of bioactive compounds in Platycladi Cacumen by UPLC-Q-TOF-MS/MS and UPLC-DAD," Journal of Pharmaceutical and Biomedical Analysis, vol. 154, pp. 207-215, 2018.

[23] P. Yang, R. Gao, and Z. Liu, "Analysis of chemical constituents and six compounds in Qu-feng-sheng-shi Granules via HPLC-ESI-Q/TOF-MS(n) and HPLC-UV technique," Biomedical Chromatography, vol. 34, no. 7, p. e4829, 2020.

[24] S. Yang, X. Zhang, Y. Dong, G. Sun, A. Jiang, and Y. Li, "Cleavage rules of mass spectrometry fragments and rapid identification of chemical components of Radix Paeoniae Alba using UHPLC-Q-TOF-MS," Phytochemical Analysis, vol. 32, no. 5, pp. 836-849, 2021.

[25] Y. Dai, Z. Dou, and R. Zhou, "Quality evaluation of artemisia capillaris thunb. Based on qualitative analysis of the HPLC fingerprint and UFLC-Q-TOF-MS/MS combined with quantitative analysis of multicomponents," Journal of Analytical Methods in Chemistry, vol. 2021, Article ID 5546446, 13 pages, 2021.

[26] H. Jin, G. Tang, J. Li, L. Ma, Y. Li, and Y. X. Chang, "Simultaneous determination of phenolic acids, anthraquinones, flavonoids, and triterpenes of cynomorii herba in different harvest times by LC-MS/MS," Journal of Analytical Methods in Chemistry, vol. 2020, Article ID 8861765, 9 pages, 2020.

[27] K. Chang, P. Gao, Y. Y. Lu, P. F. Tu, Y. Jiang, and X. Y. Guo, "Identification and characterization of quinoline alkaloids from the root bark of Dictamnus dasycarpus and their metabolites in rat plasma, urine and feces by UPLC/Qtrap-MS and UPLC/Q-TOF-MS," Journal of Pharmaceutical and Biomedical Analysis, vol. 204, Article ID 114229, 2021.

[28] Z. Ji, Y. Jiang, and H. Lin, "Global identification and quantitative analysis of representative components of Xin-NaoKang Capsule, a traditional Chinese medicinal formula, by UHPLC-Q-TOF-MS and UHPLC-TQ-MS," Journal of Pharmaceutical and Biomedical Analysis, vol. 198, Article ID 114002, 2021.
[29] M. Li, X. Yue, Y. Gao, B. Zhang, C. Yuan, and T. Wu, "Method for rapidly discovering active components in Yupingfeng granules by UPLC-ESI-Q-TOF-MS," Journal of Mass Spectrometry, vol. 55, no. 10, p. e4627, 2020.

[30] P. Lin, Q. Wang, and Y. Liu, "Characterization of chemical profile and quantification of representative components of DanLou tablet, a traditional Chinese medicine prescription, by UHPLC-Q/TOF-MS combined with UHPLC-TQ-MS," Journal of Pharmaceutical and Biomedical Analysis, vol. 180, Article ID 113070, 2020.

[31] P. Lu, Y. Chen, M. Tan, and Y. Wu, "Chemical profiling by LC-MS/MS and HPLC fingerprint combined with chemometrics and simultaneous determination of 16 characteristic ingredients for the quality consistency evaluation of ShaoyaoGancao Decoction," Biomedical Chromatography, vol. 33, no. 2, p. e4401, 2019.

[32] Q. Qian, N. Zhou, and P. Qi, “A UHPLC-QTOF-MS/MS method for the simultaneous determination of eight triterpene compounds from Poria cocos (Schw.) Wolf extract in rat plasma: application to a comparative pharmacokinetic study," J Chromatogr B Analyt Technol Biomed Life Sci, vol. 1102-1103, pp. 34-44, 2020.

[33] X. Y. Liu, Y. B. Zhang, and X. W. Yang, "Simultaneous determination of twenty-five compounds with anti-inflammatory activity in Spatholobi Caulis by using an optimized UFLC-MS/MS method: an application to pharmacokinetic study," Journal of Pharmaceutical and Biomedical Analysis, vol. 204, p. 114267, 2021.

[34] Y. Y. Lu, J. F. Chen, and J. Y. Song, "Pharmacokinetics study of 16 representative components from Baoyuan Decoction in rat plasma by LC-MS/MS with a large-volume direct injection method," Phytomedicine, vol. 57, pp. 148-157, 2019.

[35] H. Su, H. Hui, X. Xu, R. Zhou, L. Qin, and Q. Shan, "Simultaneous determination of multiple components in formula and preparations of Xiaoyaosan," Natural Product Research, vol. 35, no. 7, pp. 1207-1211, 2019.

[36] Y. L. Zhu, S. L. Li, and J. L. Jin, "Simultaneous determination of six components of Danzhi Xiaoyao Pill in beagle plasma by HPLC-MS/MS and a study of pharmacokinetic of paeoniflorin and geniposide after single-dose administration," Journal of Pharmaceutical and Biomedical Analysis, vol. 186, Article ID 113269, 2019.

[37] G. Qu, X. Qi, and S. Shao, "Pharmacokinetics of Sijunzi decoction in rat plasma after oral administration using ultra-high-performance liquid chromatography electrospray ionization quadrupole-time of flight mass spectrometry," Biomedical Chromatography, vol. 32, no. 11, p. e4334, 2019. 